\title{
On the First Eigenvalue of Bipartite Graphs
}

\author{
Amitava Bhattacharya \\ School of Mathematics \\ Tata Institute of Fundamental Research \\ Homi Bhabha Road, Colaba, Mumbai 400005, INDIA \\ amitava@math.tifr.res.in \\ Shmuel Friedland* \\ Department of Mathematics, Statistics, and Computer Science \\ University of Illinois at Chicago \\ Chicago, Illinois 60607-7045, USA \\ friedlan@uic.edu \\ Uri N. Peled \\ Department of Mathematics, Statistics, and Computer Science \\ University of Illinois at Chicago \\ Chicago, Illinois 60607-7045, USA \\ uripeled@uic.edu
}

Submitted: Sep 19, 2008; Accepted: Nov 18, 2008; Published: Nov 30, 2008

Mathematics Subject Classification: 05C07, 05C35, 05C50, 15A18

\begin{abstract}
In this paper we study the maximum value of the largest eigenvalue for simple bipartite graphs, where the number of edges is given and the number of vertices on each side of the bipartition is given. We state a conjectured solution, which is an analog of the Brualdi-Hoffman conjecture for general graphs, and prove the conjecture in some special cases.
\end{abstract}

Key words. Bipartite graph, maximal eigenvalue, Brualdi-Hoffman conjecture, degree, sequences, chain graphs.

\section{Introduction}

The purpose of this paper is to study the maximum value of the maximum eigenvalue of certain classes of bipartite graphs. These problems are analogous to the problems

${ }^{*}$ Visiting Professor, Fall 2007 - Winter 2008, Berlin Mathematical School, Berlin, Germany 
considered in the literature for general graphs and $0-1$ matrices $[1,2,3,5,8]$. We describe briefly the main problems and results obtained in this paper.

We consider only finite simple undirected graphs bipartite graphs $G$. Let $G=(V \cup$ $W, E)$, where $V=\left\{v_{1}, \ldots, v_{m}\right\}, W=\left\{w_{1}, \ldots, w_{n}\right\}$ are the two set of vertices of $G$. We view the undirected edges $E$ of $G$ as a subset of $V \times W$. Denote by $\operatorname{deg} v_{i}, \operatorname{deg} w_{j}$ the degrees of the vertices $v_{i}, w_{j}$ respectively. Let $D(G)=\left\{d_{1}(G) \geqslant d_{2}(G) \geqslant \cdots \geqslant d_{m}(G)\right\}$ be the rearranged set of the degrees $\operatorname{deg} v_{1}, \ldots, \operatorname{deg} v_{m}$. Note that $e(G)=\sum_{i=1}^{m} \operatorname{deg} v_{i}$ is the number of edges in $G$. Denote by $\lambda_{\max }(G)$ the maximal eigenvalue of $G$. Denote by $G_{\mathrm{ni}}$ the induced subgraph of $G$ consisting of nonisolated vertices of $G$. Note that $e(G)=e\left(G_{\mathrm{ni}}\right), \lambda_{\max }(G)=\lambda_{\max }\left(G_{\mathrm{ni}}\right)$. It is straightforward to show, see Proposition 2.1, that

$$
\lambda_{\max }(G) \leqslant \sqrt{e(G)}
$$

Furthermore the equality holds if and only if $G_{\mathrm{ni}}$ is a complete bipartite graph. In what follows we assume that $G=G_{\mathrm{ni}}$, unless stated otherwise.

The majority of this paper is devoted to refinements of (1.1) for noncomplete bipartite graphs. We now state the basic problem that this paper deal with. Denote by $K_{p, q}=$ $(V \cup W, E)$ the complete bipartite graph where $\# V=p, \# W=q, E=V \times W$. We assume here the normalization $1 \leq p \leqslant q$. Let $e$ be a positive integer satisfying $e \leqslant p q$. Denote by $\mathcal{K}(p, q, e)$ the family of subgraphs of $K_{p, q}$ with $e$ edges and with no isolated vertices and which are not complete bipartite graphs.

Problem 1.1. Let $2 \leqslant p \leqslant q, 1<e<p q$ be integers. Characterize the graphs which solve the maximal problem

$$
\max _{G \in \mathcal{K}(p, q, e)} \lambda_{\max }(G)
$$

We conjecture below an analog of the Brualdi-Hoffman conjecture for nonbipartite graphs [1], which was proved by Rowlinson [5]. See [3, 8] for the proof of partial cases of this conjecture.

Conjecture 1.2. Under the assumptions of Problem 1.1 an extremal graph that solves the maximal problem (1.2) is obtained from a complete bipartite graph by adding one vertex and a corresponding number of edges.

Our first result toward the solution of Problem 1.1 is of interest by itself. Let $D=$ $\left\{d_{1} \geqslant d_{2} \geqslant \cdots \geqslant d_{m}\right\}$ be a set of positive integers, and let $\mathscr{B}_{D}$ be the class of bipartite graphs $G$ with no isolated vertices, where $D(G)=D$. We show that $\max _{G \in \mathscr{B}_{D}} \lambda_{\max }(G)$ is achieved for a unique graph, up to isomorphism, which is the chain graph [9], or the difference graph [7], corresponding to $D$. (See $\S 2$.) It follows that an extremal graph solving the Problem 1.1 is a chain graph.

Our main result, Theorem 8.1, shows that Conjecture 1.2 holds in the following cases. Fix $r \geqslant 2$ and assume that $e \equiv r-1 \bmod r$. Assume that $l=\left\lfloor\frac{e}{r}\right\rfloor \geqslant r$. Let $p \in[r, l+1]$ and $q \in\left[l+1, l+1+\frac{l}{r-1}\right]$. So $K_{p, q}$ has more than $e$ edges. Then the maximum (1.2) is achieved if and only if $G$ is isomorphic to the following chain graph $G_{r, l+1}$. $G_{r, l+1}$ obtained 
from $K_{r-1, l+1}=(V \cup W, E)$ by adding an additional vertex $v_{r}$ to the set $V$, and connecting $v_{r}$ to the vertices $w_{1}, \ldots, w_{l}$ in $W$.

We now list briefly the contents of the paper. $\S 2$ is a preliminary section in which we recall some known results on bipartite graphs and related results on nonnegative matrices. In $\S 3$ we show that the maximum eigenvalue of a bipartite graph increases if we replace it by the corresponding chain graph. $\S 4$ gives upper estimates on the maximum eigenvalue of chain graphs. In $\S 5$ we discuss a minimal problem related to the sharp estimate of chain graphs with two different degrees. $\S 6$ discuses a special case of the above minimal problem

over the integers. In $\S 7$ we introduce $C$-matrices, which can be viewed as continuous analogs of the square of the adjacency matrix of chain graphs. In $\S 8$ we prove Theorem 8.1 .

\section{Preliminaries}

Figure 1: The chain graph $G_{D}$ for $D=\{5,2,2,1\}$.

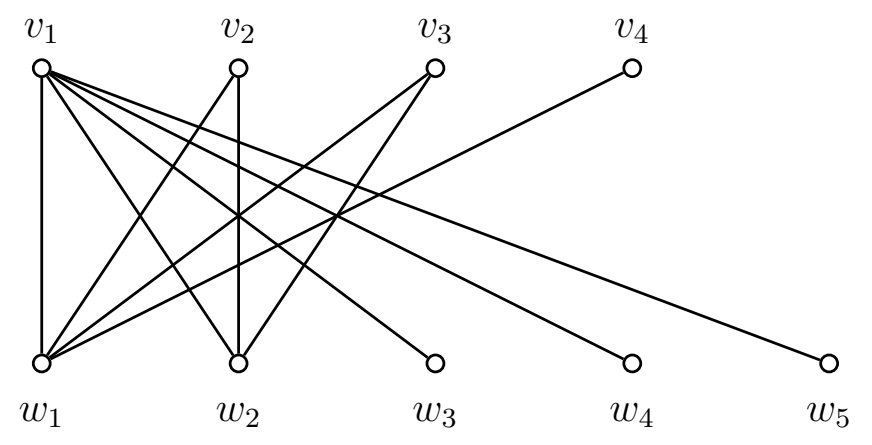

We now set up some notation and review basic results. Denote by $\mathbb{R}^{m \times n}$ the set of $m \times n$ matrices with real entries. We view $A \in \mathbb{R}^{m \times n}$ as $A=\left(A_{i, j}\right)_{i, j=1}^{m, n}$. Let $G=(V \cup W, E)$ be a bipartite graph with $V=\left\{v_{1}, \ldots, v_{m}\right\}, W=\left\{w_{1}, \ldots, w_{n}\right\}$, possibly with isolated vertices. We arrange the vertices $V \cup W$ in the order $v_{1}, \ldots, v_{m}, w_{1}, \ldots, w_{n}$. Then the adjacency matrix $B$ of $G$ is of the form

$$
B=\left(\begin{array}{cc}
0 & A \\
A^{\top} & 0
\end{array}\right)
$$

where $A$ is an $m \times n$ matrix of 0 's and 1's. We call $A$ the representation matrix of the bipartite graph $G$. Note that $i-t h$ row sum of $A$ is $\operatorname{deg} v_{i}$ and the $j-t h$ column sum of $A$ is $\operatorname{deg} w_{j}$. The graph $G$ can be specified by specifying the matrix $A$. Then $G$ does not have isolated vertices if and only if $A$ does not have zero rows and columns.

Given $D=\left\{d_{1} \geqslant d_{2} \geqslant \cdots \geqslant d_{m}\right\}$, a set of positive integers, we construct from $D$ the following graph $G_{D} \in \mathscr{B}_{D}$, well-known as a chain graph [9] or a difference graph [7]. The vertices of $G_{D}$ are partitioned into $\left\{v_{1}, \ldots, v_{m}\right\}$ and $\left\{w_{1}, \ldots, w_{n}\right\}, n=d_{1}$, and the neighbors of $v_{i}$ are $w_{1}, w_{2}, \ldots, w_{d_{i}}$. This is illustrated in Figure 1. 
We now recall the well known spectral properties of the symmetric matrix $B \in$ $\mathbb{R}^{(m+n) \times(m+n)}$ of the form (2.1), where $A \in \mathbb{R}_{+}^{m \times n}$, i.e. $A$ is $m \times n$ matrix with nonnegative entries. The spectrum of $B$ is real (by the symmetry of $B$ ) and symmetric around the origin (because if $(\mathbf{x}, \mathbf{y})$ is an eigenvector for $\lambda$, then $(\mathbf{x},-\mathbf{y})$ is an eigenvector for $-\lambda$ ). Every real matrix possesses a singular value decomposition (SVD). Specifically, if $A$ is $m \times n$ of rank $r$, then there exist positive numbers $\sigma_{i}=\sigma_{i}(A), i=1, \ldots, r$ (the singular values of $A$ ) and orthogonal matrices $U, V$ of orders $m, n$ such that $A=U \Sigma V^{\top}$, where $\Sigma=\operatorname{diag}\left(\sigma_{1}, \ldots, \sigma_{r}, 0, \ldots\right)$ is an $m \times n$ matrix having the $\sigma_{i}$ along the main diagonal and otherwise zeros. It is possible and usually done to have the $\sigma_{i}$ in non-increasing order. For symmetric matrices the singular values are the absolute values of the eigenvalues. The matrix $B$ from (2.1) satisfies $B^{2}=\left(\begin{array}{cc}A A^{\top} & A^{0} \\ 0 & A^{\top} A\end{array}\right)$, and so the eigenvalues of $B^{2}$ are those of $A A^{\top}$ together with those of $A^{\top} A$. Using the SVD for $A$ we see that $A A^{\top}$ has the $m$ eigenvalues $\sigma_{1}^{2}, \ldots, \sigma_{r}^{2}, 0,0, \ldots$ and $A^{\top} A$ has the $n$ eigenvalues $\sigma_{1}^{2}, \ldots, \sigma_{r}^{2}, 0,0, \ldots$ The eigenvalues of $B$ are therefore square roots of these numbers, and by the symmetry of the spectrum of $B$, the eigenvalues of $B$ are the $m+n$ numbers $\sigma_{1}, \ldots, \sigma_{r}, 0, \ldots, 0,-\sigma_{r}, \ldots,-\sigma_{1}$. In particular, the largest eigenvalue of $B$ is $\sigma_{1}(A)$. We denote this eigenvalue by $\lambda_{\max }(B)=\sigma_{1}(A)$. If $B$ is the adjacency matrix of $G$ then $\lambda_{\max }(G)=\lambda_{\max }(B)=\sigma_{1}(A)$.

For $\mathbf{x}=\left(x_{1}, \ldots, x_{n}\right)^{\top} \in \mathbb{R}^{n}$ we denote by $\|\mathbf{x}\|=\sqrt{\sum_{j=1}^{n} x_{j}^{2}}$, the Euclidean norm of $\mathbf{x}$. For $A \in \mathbb{R}^{m \times n}$ the operator norm of $A$ is given by $\sigma_{1}(A)=\sqrt{\lambda_{\max }\left(A A^{\top}\right)}=\sqrt{\lambda_{\max }\left(A^{\top} A\right)}$. We can find $\sigma_{1}(A)$ by the following maximum principle.

$$
\sigma_{1}(A)=\max _{\substack{\mathbf{x} \in \mathbb{R}^{m},\|\mathbf{x}\|=1 \\ \mathbf{y} \in \mathbb{R}^{n},\|\mathbf{y}\|=1}} \mathbf{x}^{\top} A \mathbf{y}=\max _{\mathbf{y} \in \mathbb{R}^{n},\|\mathbf{y}\|=1}\|A \mathbf{y}\|
$$

To see this, consider the SVD $A=U \Sigma V^{\top}$. Every $\mathbf{x} \in \mathbb{R}^{m}$ with $\|\mathbf{x}\|=1$ can be written as $\mathbf{x}=U \mathbf{a}, \mathbf{a}=\left(a_{1}, \ldots, a_{m}\right)^{\top}$, with $\|\mathbf{a}\|=1$, and every $\mathbf{y} \in \mathbb{R}^{n}$ with $\|\mathbf{y}\|=1$ can be written as $\mathbf{y}=V \mathbf{b}, \mathbf{b}=\left(b_{1}, \ldots, b_{n}\right)^{\top}$, with $\|\mathbf{b}\|=1$. Then

$$
\begin{aligned}
\mathbf{x}^{\top} A \mathbf{y}=\mathbf{a}^{\top} U^{\top} A V \mathbf{b} & =\mathbf{a}^{\top} \Sigma \mathbf{b}=\sum_{i=1}^{r} a_{i} b_{i} \sigma_{i} \leqslant \sigma_{1} \sum_{i=1}^{r}\left|a_{i} b_{i}\right| \leqslant \\
& \leqslant \sigma_{1}\left(\sum_{i=1}^{r} a_{i}^{2} \sum_{i=1}^{r} b_{i}^{2}\right)^{\frac{1}{2}} \leqslant \sigma_{1}\left(\sum_{i=1}^{m} a_{i}^{2} \sum_{i=1}^{n} b_{i}^{2}\right)^{\frac{1}{2}}=\sigma_{1}\|\mathbf{a}\|\|\mathbf{b}\|=\sigma_{1} .
\end{aligned}
$$

Equality is achieved when $\mathbf{x}$ is the first column of $U$ and $\mathbf{y}$ is the first column of $V$, and this proves the first equality of (2.2). The second equality is obtained by observing that for a given $\mathbf{y}$, the maximizing $\mathbf{x}$ is parallel to $A \mathbf{y}$.

Another useful fact that can be derived from the SVD $A=U \Sigma V^{\top}$ is the following: if $(\mathbf{x}, \mathbf{y})$ is an eigenvector of $\left(\begin{array}{rr}0 & A \\ A^{\top} & 0\end{array}\right)$ belonging to $\sigma_{1}>0$, then $\|\mathbf{x}\|=\|\mathbf{y}\|$. To see this, observe that $A \mathbf{y}=\sigma_{1} \mathbf{x}$ and $A^{\top} \mathbf{x}=\sigma_{1} \mathbf{y}$. Define vectors $\mathbf{a}=U^{\top} \mathbf{x}$ and $\mathbf{b}=V^{\top} \mathbf{y}$. Then

$$
\begin{gathered}
\Sigma \mathbf{b}=\Sigma V^{\top} \mathbf{y}=U^{\top} A \mathbf{y}=\sigma_{1} U^{\top} \mathbf{x}=\sigma_{1} \mathbf{a} \\
\Sigma^{\top} \mathbf{a}=\Sigma^{\top} U^{\top} \mathbf{x}=V^{\top} A^{\top} \mathbf{x}=\sigma_{1} V^{\top} \mathbf{y}=\sigma_{1} \mathbf{b} .
\end{gathered}
$$


It follows that for all $i$ we have $\sigma_{i} b_{i}=\sigma_{1} a_{i}$ and $\sigma_{i} a_{i}=\sigma_{1} b_{i}$. Thus $\left(\sigma_{1}+\sigma_{i}\right)\left(b_{i}-a_{i}\right)=0$. Since $\sigma_{1}+\sigma_{i}>0$, it follows that $a_{i}=b_{i}$ for all $i$, and so $\mathbf{a}=\mathbf{b}$, i.e., $U^{\top} \mathbf{x}=V^{\top} \mathbf{y}$. The orthogonal matrices $U^{\top}$ and $V^{\top}$ preserve the norms, and therefore $\|\mathbf{x}\|=\|\mathbf{y}\|$.

Recall the Rayleigh quotient characterization of the largest eigenvalue of a symmetric matric $M \in \mathbb{R}^{m \times m}: \lambda_{\max }(M)=\max _{\|\mathbf{x}\|=1} \mathbf{x}^{\top} M \mathbf{x}$. Every $\mathbf{x}$ achieving the maximum is an eigenvector of $M$ belonging to $\lambda_{\max }(M)$. If the entries of $M$ are non-negative $(M \geqslant 0)$, the maximization can be restricted to vectors $\mathbf{x}$ with non-negative entries $(\mathbf{x} \geqslant 0)$ because $\mathbf{x}^{\top} M \mathbf{x} \leqslant|\mathbf{x}|^{\top} M|\mathbf{x}|$ and $\||\mathbf{x}|\|=\|\mathbf{x}\|$, where $|\mathbf{x}|=\left(\left|x_{1}\right|, \ldots,\left|x_{m}\right|\right)^{\top}$.

Recall that a square non-negative matrix $C$ is said to be irreducible when some power of $I+C$ is positive (has positive entries). Equivalently the digraph induced by $C$ is strongly connected. Thus a symmetric non-negative matrix $B$ is irreducible when the graph induced by $B$ is connected. For a rectangular non-negative matrix $A, A A^{\top}$ is irreducible if and only if the bipartite graph with adjacency matrix $B$ given by $(2.1)$ is connected.

If a symmetric non-negative matrix $B$ is irreducible, then the Perron-Frobenius theorem implies that the spectral radius of $B$ is a simple root of the characteristic polynomial of $B$ and the corresponding eigenvector can be chosen to be positive. The following result is well known and we bring its proof for completeness.

Proposition 2.1. $A=\left(A_{i, j}\right)_{i, j=1}^{m, n} \in \mathbb{R}_{+}^{m \times n}$ and assume that $B$ is of the form (2.1). Then

$$
\lambda_{\max }(B) \leqslant \sqrt{\sum_{i, j=1}^{m, n} A_{i, j}^{2}} .
$$

Equality holds if and only if either $A=0$ or $A$ is a rank one matrix. In particular, if $G$ is a bipartite graph with $e(G) \geqslant 1$ edges then

$$
\lambda_{\max }(G) \leqslant \sqrt{e(G)}
$$

and equality holds if and only if $G_{\mathrm{ni}}$ is $K_{p, q}$, where $p q=e(G)$.

Proof. Let $r$ be the rank of $A$. Recall that the positive eigenvalues of $A A^{\top}$ are $\sigma_{1}(A)^{2}, \ldots$, $\sigma_{r}(A)^{2}$. Hence trace $A A^{\top}=\sum_{i, j}^{m, n} A_{i, j}^{2}=\sum_{k=1}^{r} \sigma_{k}(A)^{2} \geqslant \sigma_{1}(A)^{2}$. Combine this equality with the equality $\lambda_{\max }(B)=\sigma_{1}(A)$ to deduce (2.3). Clearly, equality holds if and only if either $r=0$, i.e. $A=0$, or $r=1$.

Assume now that $G$ is a bipartite graph. Let $A$ be the representation matrix of $G$. Then trace $A A^{\top}=e(G)$. Hence (2.3) implies (2.4).

Assume that $G=K_{p, q}$. Then the entries of the representation matrix $A$ consist of all 1. So rank of $A$ is one and $e\left(K_{p, q}\right)=p q$, i.e. equality holds in (2.4). Conversely, suppose that $\lambda_{\max }(G)=\sqrt{e(G)}$. Hence $\lambda_{\max }\left(G_{\mathrm{ni}}\right)=\sqrt{e\left(G_{\mathrm{ni}}\right)}$. Let $C \in \mathbb{R}^{p \times q}$ be the representation matrix of $G_{\mathrm{ni}}$. Since $G_{\mathrm{n} i}$ satisfies equality in (2.3) we deduce that $C$ is a rank one matrix. But $C$ is $0-1$ matrix that does not have a zero row or column. Hence all the rows and columns of $C$ must be identical. Hence all the entries of $C$ are 1, i.e. $G_{\mathrm{ni}}$ is a complete bipartite graph with $e(G)$ edges. 


\section{The Optimal Graphs}

The aim of this section is to prove the following theorem.

Theorem 3.1. Let $D=\left\{d_{1} \geqslant d_{2} \geqslant \cdots \geqslant d_{m}\right\}$ be a set of positive integers. Then the chain graph $G_{D}$ is the unique graph in $\mathscr{B}_{D}$, (up to isomorphism), which solves the maximum problem $\max _{G \in \mathscr{B}_{D}} \lambda_{\max }(G)$.

Let us call a graph $G \in \mathscr{B}_{D}$ optimal if it solves the maximum problem of the above theorem. Our first goal is to prove that every optimal graph is connected. For that purpose we partially order the finite sets of positive integers as follows.

Definition 3.2. Let $D=\left\{d_{1} \geqslant d_{2} \geqslant \cdots \geqslant d_{m}\right\}$ and $D^{\prime}=\left\{d_{1}^{\prime} \geqslant d_{2}^{\prime} \geqslant \cdots \geqslant d_{m^{\prime}}^{\prime}\right\}$ be sets of $m$ and $m^{\prime}$ positive integers. Then $D>D^{\prime}$ means that $m \geqslant m^{\prime}$, and $d_{1} \geqslant d_{1}^{\prime}$, $d_{2} \geqslant d_{2}^{\prime}, \ldots, d_{m^{\prime}} \geqslant d_{m^{\prime}}^{\prime}$, and $D \neq D^{\prime}$.

Theorem 3.3. If $D>D^{\prime}$, then $\lambda_{\max }\left(G_{D}\right)>\lambda_{\max }\left(G_{D^{\prime}}\right)$.

Proof. Let $A$ be the $m \times d_{1}$ matrix of 0's and 1's with row sums $d_{1} \geqslant d_{2} \geqslant \cdots \geqslant d_{m}$, and columns ordered so that each row is left-justified (1's first, then 0 's). Then $B=\left(\begin{array}{cc}0 & A \\ A^{\top} & 0\end{array}\right)$ of order $m+d_{1}$ is the adjacency matrix of $G_{D}$. Let $A^{\prime}$ and $B^{\prime}$ be defined similarly for $G_{D^{\prime}}$.

Let $M=B B^{\top}$ and $M^{\prime}=B^{\prime} B^{\prime \top}$. Then $M$ and $M^{\prime}$ are symmetric non-negative irreducible matrices of orders $m$ and $m^{\prime}$, and $\lambda_{\max }\left(G_{D}\right)=\lambda_{\max }(M), \lambda_{\max }\left(G_{M^{\prime}}\right)=\lambda_{\max }\left(M^{\prime}\right)$.

Case 1: $m=m^{\prime}$. In this case, by Definition 3.2, at least one of the inequalities $d_{1} \geqslant d_{1}^{\prime}$, $d_{2} \geqslant d_{2}^{\prime}, \ldots, d_{m} \geqslant d_{m}^{\prime}$ holds with strict inequality. It follows that $M \geqslant M^{\prime}$ (i.e., $M-M^{\prime}$ is a non-negative matrix), and some integer $i \in[1, m]$ satisfies $M_{i, i}>M_{i, i}^{\prime}$. Therefore every positive vector $\mathbf{y}$ satisfies $\mathbf{y}^{\top} M \mathbf{y}>\mathbf{y}^{\top} M^{\prime} \mathbf{y}$. Let $\mathbf{y}=\mathbf{x}^{\prime}$ be the positive Perron-Frobenius eigenvector of the irreducible matrix $M^{\prime}$, with $\left\|\mathbf{x}^{\prime}\right\|=1$. Then by the Rayleigh quotient we have

$$
\lambda_{\max }(M) \geqslant \mathbf{x}^{\prime \top} M \mathbf{x}^{\prime}>\mathbf{x}^{\prime \top} M^{\prime} \mathbf{x}^{\prime}=\lambda_{\max }\left(M^{\prime}\right),
$$

as required.

Case 2: $m>m^{\prime}$. In this case, let $L$ be the principal submatrix of $M$ consisting of its first $m^{\prime}$ rows and columns. By Definition 3.2 we have $d_{1} \geqslant d_{1}^{\prime}, d_{2} \geqslant d_{2}^{\prime}, \ldots, d_{m^{\prime}} \geqslant d_{m^{\prime}}^{\prime}$. Therefore $L \geqslant M^{\prime}$, and hence $\lambda_{\max }(L) \geqslant \lambda_{\max }\left(M^{\prime}\right)$.

Since $L$ is symmetric and non-negative, there exists a vector $\mathbf{y} \in \mathbb{R}^{m^{\prime}}$ with $\mathbf{y} \geqslant \mathbf{0}$, $\|\mathbf{y}\|=1$ satisfying $\lambda_{\max }(L)=\mathbf{y}^{\top} L \mathbf{y}$. Extend $\mathbf{y}$ with zeros to a vector $\mathbf{x} \in \mathbb{R}^{m}$. Then $\mathbf{x} \geqslant \mathbf{0}$ and $\|\mathbf{x}\|=1$ and $\mathbf{y}^{\top} L \mathbf{y}=\mathbf{x}^{\top} M \mathbf{x} \leqslant \lambda_{\max }(M)$. Equality cannot occur here, for if it did, then $\mathbf{x}$ would be the unique Perron-Frobenius eigenvector of the irreducible matrix $M$ and $\mathbf{x}$ would be positive, whereas $x_{i}=0$ for $i>m^{\prime}$. Thus $\lambda_{\max }(M)>\lambda_{\max }(L) \geqslant \lambda_{\max }\left(M^{\prime}\right)$, as required.

Lemma 3.4. If $G \in \mathscr{B}_{D}$ is connected, then $\lambda_{\max }(G) \leqslant \lambda_{\max }\left(G_{D}\right)$. 
Proof. Let $D=\left\{d_{1} \geqslant \cdots \geqslant d_{m}\right\}$ and $n \geqslant d_{1}$. Let $B=\left(\begin{array}{cc}0 & A \\ A^{\top} & 0\end{array}\right)$ be the adjacency matrix of $G$, where $A$ is $m \times n$ with row sums given by $D$. Since $G$ is connected, $B$ is irreducible. Let $(\mathbf{x}, \mathbf{y})$ be the positive Perron-Frobenius eigenvector of $B$ belonging to $\lambda_{\max }(G)=\sigma_{1}(A)$, with $\mathbf{x}=\left(x_{1}, \ldots, x_{m}\right), \mathbf{y}=\left(y_{1}, \ldots, y_{n}\right)$ :

$$
\sigma_{1}(A)\left(\begin{array}{l}
\mathbf{x} \\
\mathbf{y}
\end{array}\right)=\left(\begin{array}{cc}
0 & A \\
A^{\top} & 0
\end{array}\right)\left(\begin{array}{l}
\mathbf{x} \\
\mathbf{y}
\end{array}\right)
$$

and so $A \mathbf{y}=\sigma_{1}(A) \mathbf{x}$.

As we observed in Section 2, we have $\|\mathbf{x}\|=\|\mathbf{y}\|$, and so we may choose a normalization such that $\|\mathbf{x}\|=\|\mathbf{y}\|=1$.

We reorder the columns of $A$ so that $y_{1} \geqslant y_{2} \geqslant \cdots \geqslant y_{n}>0$. The rows are still in their original order, and so the row sums are $d_{1} \geqslant \cdots \geqslant d_{m}$ in this order.

Let $\overleftarrow{A}$ be the matrix obtained from $A$ by left-justifying each row, i.e., moving all the

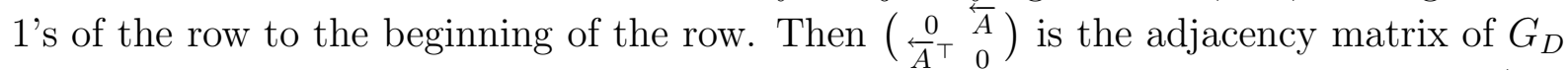
with $n-d_{1}$ zero rows and columns appended at the end, and therefore $\lambda_{\max }\left(G_{D}\right)=\sigma_{1}(\overleftarrow{A})$

Since $y_{1} \geqslant y_{2} \geqslant \cdots \geqslant y_{n} \geqslant 0$ and since $\overleftarrow{A}$ is obtained from $A$ by left-justifying each row, we have $\overleftarrow{A} \mathbf{y} \geqslant A \mathbf{y}$. Since $\mathbf{x} \geqslant \mathbf{0}$, we have $\mathbf{x}^{\top} \overleftarrow{A} \mathbf{y} \geqslant \mathbf{x}^{\top} A \mathbf{y}$. (2.2) yields

$$
\begin{aligned}
\lambda_{\max }\left(G_{D}\right)=\sigma_{1}(\overleftarrow{A})=\max _{\substack{\mathbf{u} \in \mathbb{R}^{m},\|\mathbf{u}\|=1 \\
\mathbf{v} \in \mathbb{R}^{n},\|\mathbf{v}\|=1}} \mathbf{u}^{\top \overleftarrow{A} \mathbf{v} \geqslant \mathbf{x}^{\top \overleftarrow{A}} \mathbf{y} \geqslant \mathbf{x}^{\top} A \mathbf{y}} \\
\quad=\mathbf{x}^{\top} \sigma_{1}(A) \mathbf{x}=\sigma_{1}(A)=\lambda_{\max }(G)
\end{aligned}
$$

Lemma 3.5. An optimal graph must be connected.

Proof. Let $G \in \mathscr{B}_{D}$ be an optimal graph. The graph $G$ is bipartite, and one side of the bipartition (call it the first side) has degrees given by $D$. Let $G_{1}, \ldots, G_{k}$ be the connected components of $G$. Then $\lambda_{\max }(G)=\lambda_{\max }\left(G_{i}\right)$ for some $i$.

Like $G$, the component $G_{i}$ is also bipartite with the bipartition inherited from that of $G$. Let $D_{i}$ be the set of degrees of $G_{i}$ on the first side of the bipartition.

If $G$ is disconnected, then $D>D_{i}$, and therefore $\lambda_{\max }(G) \geqslant \lambda_{\max }\left(G_{D}\right)>\lambda_{\max }\left(G_{D_{i}}\right) \geqslant$ $\lambda_{\max }\left(G_{i}\right)$, where the first inequality is by the optimality of $G$, the second by Theorem 3.3, and the third by Lemma 3.4 and the connectivity of $G_{i}$. This contradicts the equality above and proves that $G$ must be connected.

We are now ready to prove our main theorem.

Proof. (of Theorem 3.1). Let $G \in \mathscr{B}_{D}$ be optimal with adjacency matrix $B=\left(\begin{array}{cc}0 & A \\ A^{\top} & 0\end{array}\right)$. By Lemma 3.5 $G$ is connected. We begin as in the proof of Lemma 3.4. We let $(\mathbf{x}, \mathbf{y})$ be the positive Perron-Frobenius eigenvector of $B$ belonging to $\lambda_{\max }(G)=\sigma_{1}(A)$, with $\mathbf{x}=\left(x_{1}, \ldots, x_{m}\right)^{\top}, \mathbf{y}=\left(y_{1}, \ldots, y_{n}\right)^{\top},\|\mathbf{x}\|=\|\mathbf{y}\|=1$. In other words, (3.1) holds, or equivalently

$$
\begin{aligned}
A \mathbf{y} & =\sigma_{1}(A) \mathbf{x} \\
A^{\top} \mathbf{x} & =\sigma_{1}(A) \mathbf{y}
\end{aligned}
$$


However, this time we reorder both the rows and the columns of $A$ so that $x_{1} \geqslant x_{2} \geqslant$ $\cdots \geqslant x_{m}>0$ and $y_{1} \geqslant y_{2} \geqslant \cdots \geqslant y_{n}>0$, so now the row sums of $A$, which we still denote by $d_{1}, d_{2}, \ldots, d_{m}$, are not necessarily non-decreasing. As before, we let $\overleftarrow{A}$ be the matrix obtained from $A$ by left-justifying each row. The graph with adjacency matrix $\left(\begin{array}{cc}\frac{0}{A^{\top}} & \overleftarrow{A} \\ A^{-}\end{array}\right)$is still isomorphic to $G_{D}$ plus $n-d_{1}$ isolated vertices, and therefore $\lambda_{\max }\left(G_{D}\right)=\sigma_{1}(\overleftarrow{A})$. For the same reasons as before we have $\overleftarrow{A} \mathbf{y} \geqslant A \mathbf{y}$, and therefore (3.2) holds. Moreover, by the optimality of $G$ we have equality throughout (3.2). In particular $G_{D}$ is optimal and $\lambda_{\max }\left(G_{D}\right)=\sigma_{1}(A)$, so from now on we abbreviate $\sigma_{1}(A)=\sigma_{1}(\overleftarrow{A})=\sigma_{1}$. Now $\overleftarrow{A} \mathbf{y} \geqslant A \mathbf{y}$ and $\mathbf{x}^{\top} \overleftarrow{A} \mathbf{y}=\mathbf{x}^{\top} A \mathbf{y}$ and $\mathbf{x}>\mathbf{0}$ give

$$
\overleftarrow{A} \mathbf{y}=A \mathbf{y}=\sigma_{1} \mathbf{x}
$$

The first two rows of (3.5) and $x_{1} \geqslant x_{2}$ now give

$$
y_{1}+\cdots+y_{d_{1}}=\sigma_{1} x_{1} \geqslant \sigma_{1} x_{2}=y_{1}+\cdots+y_{d_{2}},
$$

and since $\mathbf{y}>\mathbf{0}$ we must have $d_{1} \geqslant d_{2}$. The same argument with rows 2 and 3 shows $d_{2} \geqslant d_{3}$, and so on. We have established that the row sums of $A$ are non-decreasing, i.e.,

$$
d_{1} \geqslant d_{2} \geqslant \cdots \geqslant d_{m}
$$

Note that by (3.6), the columns of $\overleftarrow{A}$ are top-justified, i.e., the 1's are above the 0's. For this reason and $\mathbf{x} \geqslant \mathbf{0}$ we have $\overleftarrow{A}^{\top} \mathbf{x} \geqslant A^{\top} \mathbf{x}$, and hence $\mathbf{y}^{\top} \overleftarrow{A}^{\top} \mathbf{x} \geqslant \mathbf{y}^{\top} A^{\top} \mathbf{x}$ by $\mathbf{y} \geqslant \mathbf{0}$ The analog of (3.2) for $\overleftarrow{A}^{\top}$ now holds with equality throughout and we obtain

$$
\overleftarrow{A}^{\top} \mathbf{x}=A^{\top} \mathbf{x}=\sigma_{1} \mathbf{y}
$$

Our remaining task is to show that $d_{1}=n$ and $A=\overleftarrow{A}$, and therefore $G$ is isomorphic to $G_{D}$. For that purpose we need notation for rows of $\overleftarrow{A}$ with equal sums, and similarly for columns.

We introduce the following notation for the row sums of $\overleftarrow{A}$

$$
\begin{array}{r}
r_{1}=d_{1}=\cdots=d_{m_{1}}>r_{2}=d_{m_{1}+1}=\cdots=d_{m_{1}+m_{2}}>\cdots> \\
>r_{h}=d_{m_{1}+\cdots+m_{h-1}+1}=\cdots=d_{m_{1}+\cdots+m_{h}},
\end{array}
$$

where

$$
m_{1}+\cdots+m_{h}=m .
$$

This is illustrated in Figure 2 .

From (3.5) we have $\sigma_{1} x_{i}=(\overleftarrow{A} \mathbf{y})_{i}=y_{1}+\cdots+y_{d_{i}}$. Therefore by (3.8) and $\mathbf{y}>\mathbf{0}$ we obtain

$$
\begin{array}{r}
x_{1}=\cdots=x_{m_{1}}>x_{m_{1}+1}=\cdots=x_{m_{1}+m_{2}}>\cdots> \\
x_{m_{1}+\cdots+m_{h-1}+1}=\cdots=x_{m_{1}+\cdots+m_{h}}>0 .
\end{array}
$$


Figure 2: The notation for the row sums of $\overleftarrow{A}$

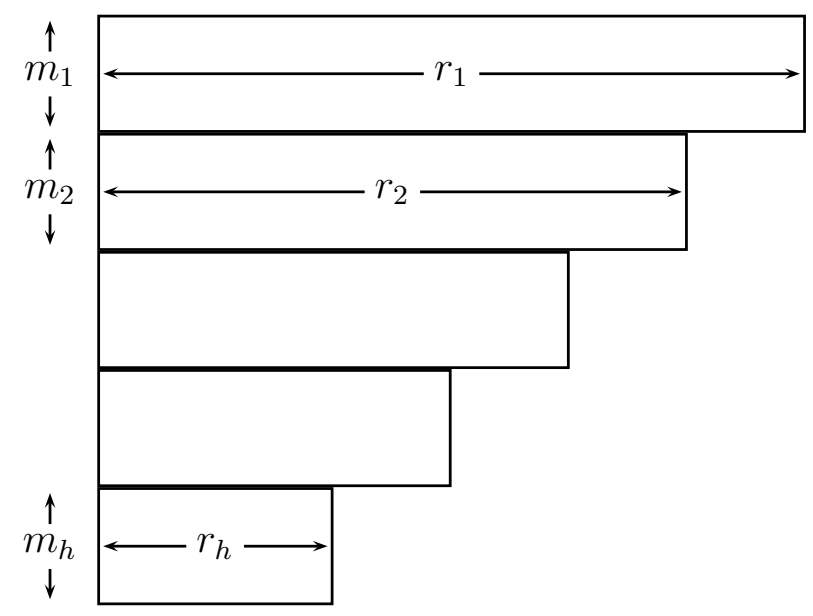

Analogously using (3.7) and (3.8) and $\mathbf{x}>\mathbf{0}$ we obtain

$$
\begin{array}{r}
y_{1}=\cdots=y_{r_{h}}>y_{r_{h}+1}=\cdots=y_{r_{h-1}}>\cdots> \\
y_{r_{2}+1}=\cdots=y_{r_{1}}>0=y_{r_{1}+1}=y_{r_{1}+2}=\cdots=y_{n} .
\end{array}
$$

From (3.10) and $\mathbf{y}>\mathbf{0}$, we conclude that

$$
d_{1}=r_{1}=n
$$

We are now ready to show that $A=\overleftarrow{A}$. Since $d_{1}=r_{1}=n$, the first $m_{1}$ rows of $A$ are all-1, and so are the first $m_{1}$ rows of $\overleftarrow{A}$. Now let $m_{1}+1 \leqslant i \leqslant m_{1}+m_{2}$ be an index of one of the next $m_{2}$ rows. Both $A$ and $\overleftarrow{A}$ have $d_{i}=r_{2} 1$ 's in row $i$. Let the 1 's in row $i$ of $A$ lie in columns $k_{1}, \ldots, k_{r_{2}}$. Then by $(3.5)$ we have

$$
\sum_{j=1}^{r_{2}} y_{k_{j}}=(A \mathbf{y})_{i}=(\overleftarrow{A} \mathbf{y})_{i}=\sum_{j=1}^{r_{2}} y_{j}
$$

However, by (3.10) the last $r_{1}-r_{2}$ components of $\mathbf{y}$ are smaller than all other components. Therefore if any $k_{j}$ lies in the range $\left\{r_{2}+1, \ldots, r_{1}\right\}$, it would follow that $\sum_{j=1}^{r_{2}} y_{k_{j}}<$ $\sum_{j=1}^{r_{2}} y_{j}$, contradicting (3.11). Therefore $k_{j}=j$ for $j=1 \ldots, r_{2}$, in other words rows $i$ of $A$ and $\overleftarrow{A}$ are the same.

An analogous argument can be applied to the next $m_{3}$ rows, and so on, and it follows that $\overleftarrow{A}=A$

The arguments of the proof of the above theorem yield.

Corollary 3.6. Let the assumptions of Problem 1.1 holds. Then any $H \in \mathcal{K}(p, q, e)$ satisfying $\max _{G \in \mathcal{K}(p, q, e)} \lambda_{\max }(G)=\lambda_{\max }(H)$ is isomorphic to $G_{D}$, for some $D=\left\{d_{1} \geqslant\right.$ $\left.d_{2} \geqslant \cdots \geqslant d_{m}\right\}$, where $m \leqslant p$ and $d_{1} \leqslant q$. 


\section{Estimations of the Largest Eigenvalue}

In this section we give lower and upper bounds for $\lambda_{\max }(G)$, where $G$ is an optimal graph with a given adjacency matrix $\left(\begin{array}{cc}0 & A \\ A^{\top} & 0\end{array}\right)$. (Our upper bound improves the upper bound (2.4).)

Recall the concept of the second compound matrix $\Lambda_{2} A$ of an $m \times n$ matrix $A=\left(A_{i, j}\right)$ [6]: $\Lambda_{2} A$ is an $\left(\begin{array}{c}m \\ 2\end{array}\right) \times\left(\begin{array}{c}n \\ 2\end{array}\right)$ matrix with rows indexed by $\left(i_{1}, i_{2}\right), 1 \leqslant i_{1}<i_{2} \leqslant m$ and columns indexed by $\left(j_{1}, j_{2}\right), 1 \leqslant j_{1}<j_{2} \leqslant n$. The entry in row $\left(i_{1}, i_{2}\right)$ and column $\left(j_{1}, j_{2}\right)$ of $\Lambda_{2} A$ is given by

$$
\Lambda_{2} A_{\left(i_{1}, i_{2}\right)\left(j_{1}, j_{2}\right)}=\operatorname{det}\left(\begin{array}{c}
A_{i_{1}, j_{1}} A_{i_{1}, j_{2}} \\
A_{i_{2}, j_{1}} A_{i_{2}, j_{2}}
\end{array}\right) .
$$

Note that $\left(\Lambda_{2} A\right)^{\top}=\Lambda_{2} A^{\top}$. It follows from the Cauchy-Binet theorem that for matrices $A, B$ of compatible dimensions one has $\Lambda_{2}(A B)=\left(\Lambda_{2} A\right)\left(\Lambda_{2} B\right)$. One also has $\Lambda_{2} I=I$ and therefore $\Lambda_{2} A^{-1}=\left(\Lambda_{2} A\right)^{-1}$ for nonsingular $A$. In particular, the second compound matrix of an orthogonal matrix is orthogonal, and therefore the SVD carries over to the second compound: if the SVD of $A$ is $A=U \Sigma V^{\top}$, then the SVD of $\Lambda_{2} A$ is $\left(\Lambda_{2} A\right)=$ $\left(\Lambda_{2} U\right)\left(\Lambda_{2} \Sigma\right)\left(\Lambda_{2} V\right)^{\top}$. It follows that if the singular values of $A$ are $\sigma_{1} \geqslant \sigma_{2} \geqslant \cdots$, then the singular values of $\Lambda_{2} A$ are $\sigma_{i} \sigma_{j}, i<j$. In particular, when the rank of $A$ is larger than 1 , equivalently $\Lambda_{2} A \neq 0$, we have

$$
\sigma_{1} \sigma_{2}=\sigma_{1}\left(\Lambda_{2} A\right)=\max _{\mathbf{w} \neq \mathbf{0}} \frac{\left\|\left(\Lambda_{2} A\right) \mathbf{w}\right\|}{\|\mathbf{w}\|},
$$

where the second equality follows by applying (2.2) to $\Lambda_{2} A$.

We now specialize to $A$ given by (2.1), which is the adjacency matrix of an optimal graph. Thus $A$ is a matrix of 0's and 1's whose rows are left-justified and whose columns are top-justified. We use the notation (3.8) for the row sums of $A$. For such $A$ the entries of $\Lambda_{2} A$ can only be 0 or -1 . Indeed, if in (4.1) $A_{i_{2}, j_{2}}=1$, then $A_{i_{1}, j_{2}}=A_{i_{2}, j_{1}}=A_{i_{1}, j_{1}}=1$ and the determinant vanishes. If $A_{i_{2}, j_{2}}=0$ and the determinant does not vanish, then again $A_{i_{1}, j_{2}}=A_{i_{2}, j_{1}}=A_{i_{1}, j_{1}}=1$ and the determinant equals -1 . In the latter case we say that $\left(i_{1}, i_{2}\right)$ and $\left(j_{1}, j_{2}\right)$ are in a $\Gamma$-configuration.

To estimate $\sigma_{1} \sigma_{2}$ from below, we take a particular column vector $\mathbf{w}$ in (4.2): the $\left(j_{1}, j_{2}\right), j_{1}<j_{2}$ entry of $\mathbf{w}$ is 1 if column $\left(j_{1}, j_{2}\right)$ of $\Lambda_{2} A$ is nonzero; otherwise this entry of $\mathbf{w}$ is zero. (The assumption $\Lambda_{2} A \neq 0$ implies that $\mathbf{w} \neq \mathbf{0}$.) By $(4.2)$ we have

$$
\sigma_{1} \sigma_{2} \geqslant \frac{\left\|\left(\Lambda_{2} A\right) \mathbf{w}\right\|}{\|\mathbf{w}\|} .
$$

Since $\mathbf{w}$ is a vector of 0 's and 1's, $\|\mathbf{w}\|^{2}$ is the number of nonzero entries of $\mathbf{w}$, that is to say, the number of nonzero columns of $\Lambda_{2} A$. We count the nonzero columns $\left(j_{1}, j_{2}\right), j_{1}<j_{2}$ of $\Lambda_{2} A$ as follows. Fix $j_{2}$. There is a unique $k=1, \ldots, h-1$ such that $r_{k+1}+1 \leqslant j_{2} \leqslant r_{k}$. If $j_{1}$ is chosen among $1, \ldots, r_{k+1}$, then there exist $\left(i_{1}, i_{2}\right)$ such that $\left(i_{1}, i_{2}\right)$ and $\left(j_{1}, j_{2}\right)$ are in a $\Gamma$-configuration, and otherwise not. It follows that for our fixed $j_{2}$, there are $r_{k+1}$ values of $j_{1}$ such that column $\left(j_{1}, j_{2}\right)$ of $\Lambda_{2} A$ is nonzero. We can vary $j_{2}$ without changing 
$k$ in $r_{k}-r_{k+1}$ ways, so $\Lambda_{2} A$ has $r_{k+1}\left(r_{k}-r_{k+1}\right)$ nonzero columns corresponding to the same $k$. Summing over $k$, we conclude that

$$
\|\mathbf{w}\|^{2}=\sum_{k=1}^{h-1} r_{k+1}\left(r_{k}-r_{k+1}\right) .
$$

By similar arguments we see that for $1 \leqslant k<l \leqslant h$, the vector $\left(\Lambda_{2} A\right) \mathbf{w}$ has $m_{k} m_{l}$ entries equal to $-r_{l}\left(r_{k}-r_{l}\right)$, and that all other entries of $\left(\Lambda_{2} A\right) \mathbf{w}$ vanish. Therefore

$$
\left\|\left(\Lambda_{2} A\right) \mathbf{w}\right\|^{2}=\sum_{1 \leqslant k<l \leqslant h} m_{k} m_{l}\left[r_{l}\left(r_{k}-r_{l}\right)\right]^{2} .
$$

From (4.3), (4.4) and (4.5), we obtain

$$
\sigma_{1}^{2} \sigma_{2}^{2} \geqslant \omega \equiv \frac{\sum_{1 \leqslant k<l \leqslant h} m_{k} m_{l}\left[r_{l}\left(r_{k}-r_{l}\right)\right]^{2}}{\sum_{k=1}^{h-1} r_{k+1}\left(r_{k}-r_{k+1}\right)} .
$$

As we have noted above, we assume that $h>1$, for otherwise $A$ has rank 1 . If $h=1$ we define $\omega=0$.

We can improve the lower bound (4.6) by the following consideration. The graph with adjacency matrix $\left(\begin{array}{cc}0 & A^{\top} \\ A & 0\end{array}\right)$ is isomorphic to the one with adjacency matrix $\left(\begin{array}{cc}0 & A \\ A^{\top} & 0\end{array}\right)$. Therefore we can repeat the work in this section with $A^{\top}$ replacing $A$. This amounts to transposing the Ferrers diagram illustrated in Figure 2. Instead of (4.6) we now have

$$
\sigma_{1}^{2} \sigma_{2}^{2} \geqslant \omega^{\prime} \equiv \frac{\sum_{1 \leqslant k<l \leqslant h} m_{k}^{\prime} m_{l}^{\prime}\left[r_{l}^{\prime}\left(r_{k}^{\prime}-r_{l}^{\prime}\right)\right]^{2}}{\sum_{k=1}^{h-1} r_{k+1}^{\prime}\left(r_{k}^{\prime}-r_{k+1}^{\prime}\right)}
$$

where for $i=1, \ldots, h$ we have $r_{i}^{\prime}=m_{1}+\cdots+m_{h-i+1}$ and $m_{i}^{\prime}=r_{h-i+1}-r_{h-i+2}\left(r_{h+1}=0\right)$.

Combining (4.6) and (4.7) we obtain

$$
\sigma_{1}^{2} \sigma_{2}^{2} \geqslant \omega^{*} \equiv \max \left\{\omega, \omega^{\prime}\right\}
$$

We are now ready to estimate $\sigma_{1}^{2}$ from above.

Theorem 4.1. Let $D=\left\{d_{1} \geqslant d_{2} \geqslant \cdots \geqslant d_{m}\right\}$ be a set of positive integers, where $d_{1}>d_{m}$. Then

$$
\lambda_{\max }\left(G_{D}\right)^{2} \leqslant \frac{e\left(G_{D}\right)+\sqrt{e\left(G_{D}\right)^{2}-4 \omega^{*}\left(G_{D}\right)}}{2},
$$

where $\omega^{*}\left(G_{D}\right)$ is defined in (4.8). Assume that in the Ferrers diagram given in Figure 2 $h=2$. I.e. the degree of the vertices in each group of $G_{D}$ have exactly two distinct values. Then equalities hold in (4.6), (4.7), (4.8) and (4.9). In particular

$$
\omega^{*}(D)=\omega=\omega^{\prime}=m_{1} m_{2} r_{2}\left(r_{1}-r_{2}\right) .
$$


Proof. Since the eigenvalues of $A^{\top} A$ are $\lambda_{\max }\left(G_{D}\right)^{2}=\sigma_{1}^{2}, \sigma_{2}^{2}, \ldots, \sigma_{r}^{2}, 0,0, \ldots$, we have $\sum_{i=1}^{r} \sigma_{i}^{2}=\operatorname{trace} A^{\top} A=\sum_{i, j} A_{i, j}^{2}=e$. Let us denote $a=\sigma_{1}^{2}+\sigma_{2}^{2}$, so that $a \leqslant e=e\left(G_{D}\right)$, and $b=\sigma_{1}^{2} \sigma_{2}^{2}$, so that $b \geqslant \omega^{*}$ by (4.8). Solving for $\sigma_{1}^{2}$ we obtain $\sigma_{1}^{2}=\frac{a+\sqrt{a^{2}-4 b}}{2} \leqslant \frac{e+\sqrt{e^{2}-4 \omega^{*}}}{2}$.

Assume that $h=2$. Then $A$ has rank $2, \Lambda_{2} A$ has rank 1 , and $a=e$. Furthermore the definitions of $\omega, \omega^{\prime}$ yield the equalities (4.10). To complete the proof, we show that equality holds in (4.3) and therefore also in (4.6), i.e., $b=\omega$.

Since $\Lambda_{2} A$ has rank 1 and its elements are only 0 and -1 , all its nonzero rows are equal. Say it has $c$ nonzero rows, each with $d$ elements of -1 . The trace of $\left(\Lambda_{2} A\right)^{\top}\left(\Lambda_{2} A\right)$ is the sum of squares of the singular values of $\Lambda_{2} A$, which equals $\left(\sigma_{1}\left(\Lambda_{2} A\right)\right)^{2}=\sigma_{1}^{2} \sigma_{2}^{2}$ in our case. This trace also equals the sum of squares of the elements of $\Lambda_{2} A$, namely $c d$.

On the other hand, our chosen vector $\mathbf{w}$ satisfies $\|\mathbf{w}\|^{2}=d$ and $\left\|\left(\Lambda_{2} A\right) \mathbf{w}\right\|^{2}=c d^{2}$ (because each of the $c$ nonzero rows of $\Lambda_{2} A$ multiplied by $\mathbf{w}$ gives $-d$ ). Hence $\frac{\left\|\left(\Lambda_{2} A\right) \mathbf{w}\right\|^{2}}{\|\mathbf{w}\|^{2}}=$ $c d$. Thus both sides of (4.3) are equal to $\sqrt{c d}$.

We suspect that under the conditions of Theorem 4.1 for $h \geqslant 3$ one has strict inequality in $(4.9)$.

\section{A Minimization Problem}

The first step in proving Conjecture 1.2 is to show its validity in the case $h=2$ in Figure 2 . We note that for $h=2$, (4.9) is tight by Theorem 4.1. Theorem 4.1 also implies that equality holds in (4.6), (4.7) and (4.8). This motivates us to consider the problem of minimizing $\omega^{*}(G)$.

Let $n_{1}:=r_{2}, n_{2}:=r_{1}-r_{2}$. Then the condition that the chain graph $G$ has $e$ edges is equivalent to

$$
m_{1} n_{1}+m_{1} n_{2}+m_{2} n_{1}=e .
$$

Formula (4.6) for the case $h=2$ gives

$$
\omega=m_{1} m_{2} n_{1} n_{2} .
$$

Let $\mathcal{K}_{2}(p, q, e) \subseteq \mathcal{K}(p, q, e)$ be set of all subgraphs of $K_{p, q}$ isomorphic to some $G_{D}$ whose Ferrers diagram, given in Figure 2, satisfies the condition $h=2$. By the above discussion for $h=2$, the problem of finding $\max _{G \in \mathcal{K}_{2}(p, q, e)} \lambda_{\max }(G)$ is equivalent to the following minimization problem over the integers.

Problem 5.1. Let $p, q$ and $e$ be integers satisfying $2 \leqslant p \leqslant q$ and $3 \leqslant e<p q$. Find the minimum of $m_{1} m_{2} n_{1} n_{2}$ in positive integers $m_{1}, m_{2}, n_{1}$ and $n_{2}$ satisfying $m_{1}+m_{2} \leqslant p$, $n_{1}+n_{2} \leqslant q$ and the constraint (5.1).

Note if $p=q$, then Problem 5.1 remains invariant under the duality of exchanging $\left(m_{1}, m_{2}\right)$ with $\left(n_{1}, n_{2}\right)$. Conjecture 1.2 implies that any minimal solution of Problem 5.1 satisfies the condition $\min \left(m_{2}, n_{2}\right)=1$. 
In order to prove Conjecture 1.2 in the cases discussed in Theorem 8.1 we need to consider a problem of minimizing $m_{1} m_{2} n_{1} n_{2}$ under certain constraints, where $m_{1}, m_{2}, n_{1}$ and $n_{2}$ are real numbers. We start with the following simple lemma.

Lemma 5.2. Let $a, b$ and $e$ be positive real numbers satisfying $e>a+b$. Assume that

$$
a x+b y=e, \quad 1 \leqslant x, 1 \leqslant y .
$$

Then

$$
x y \geqslant \min \left(\frac{e-a}{b}, \frac{e-b}{a}\right) .
$$

Equality holds if and only if

1. $x=1$ when $a<b$;

2. $y=1$ when $b<a$;

3. $x=1$ or $y=1$ when $a=b$.

Proof. Set $b y=e-a x$ and observe that $f(x):=b y x=(e-a x) x$. Note that $f$ is a parabola, with its maximum at $x_{0}:=\frac{e}{2 a}$. So $f$ is decreasing for $x>x_{0}$ and increasing for $x<x_{0}$. The minimum of $x y=\frac{f}{b}$, given the constraints $x \geqslant 1, y \geqslant 1$ is achieved only when $x=1$ and $y=\frac{e-a}{b}>1$ (the minimum possible value of $x$ ), or when $y=1$ and $x=\frac{e-b}{a}>1$ (where $x$ is the maximum possible value). For $a<b$ and $x=1$ we have $x y=\frac{a-a}{b}$, which is the LHS of (5.4). For $b<a$ and $y=1$ we have $x y=\frac{e-b}{a}$, which is the RHS of (5.4).

Proposition 5.3. Let $2 \leqslant r, e \in \mathbb{N}$ and assume that

$$
e=l r+r-1, \quad r \leqslant p, q \leqslant l+1+\frac{l}{r-1} .
$$

Let $G=(V, W, E) \in \mathcal{K}(p, q, e)$. Then $\# V \geqslant r$ and $\# W \geqslant r$.

Proof. Assume to the contrary that $\# V \leqslant r-1$. Since $G$ is not a complete bipartite graph,

$$
e<(r-1) q \leqslant(r-1)\left(l+1+\frac{l}{r-1}\right)=l r+r-1=e,
$$

which is impossible. Replacing $q$ by $p$ we deduce that $\# W \geqslant r$.

Hence if $p, q$ and $e$ satisfy (5.5), then for $D \in \mathcal{K}_{2}(p, q, e)$ we must have $m_{1}+m_{2} \geqslant r$ and $n_{1}+n_{2} \geqslant r$. Since $m_{1}, m_{2}, n_{1}, n_{2}$ are positive integers, they satisfy the following constraints

$$
m_{\geqslant} \geqslant 1, m_{2} \geqslant 1, n_{1} \geqslant 1, n_{2} \geqslant 1, m_{1}+m_{2} \geqslant r, n_{1}+n_{2} \geqslant r .
$$


Theorem 5.4. Let $2 \leqslant r \in \mathbb{N}, e \in\left[r^{2}+1, \infty\right)$ and consider the minimum of $\omega=m_{1} m_{2} n_{1} n_{2}$ subject to $m_{1}, m_{2}, n_{1}, n_{2} \in \mathbb{R}$, (5.1) and (5.6). Then the minimum is $\frac{(r-1)(e-r+1)}{r}$, and it is achieved only in one of the two cases

$$
\begin{aligned}
& \left(m_{1}, m_{2}\right)=(r-1,1),\left(n_{1}, n_{2}\right)=\left(\frac{e-r+1}{r}, 1\right) \\
& \left(m_{1}, m_{2}\right)=\left(\frac{e-r+1}{r}, 1\right),\left(n_{1}, n_{2}\right)=(r-1,1) .
\end{aligned}
$$

Proof. Let $\bar{\omega}$ be the minimum value of $\omega$ subject (5.1) and (5.6). Note that since for the values of $m_{1}, m_{2}, n_{1}, n_{2}$ given in (5.7) we have $\omega=\frac{(r-1)(e-r+1)}{r}$, we deduce that $\bar{\omega} \leqslant$ $\frac{(r-1)(e-r+1)}{r}$. Since all the functions are symmetric in $\left(m_{1}, m_{2}\right)$ and $\left(n_{1}, n_{2}\right)$, we will always assume that $m_{1}+m_{2} \leqslant n_{1}+n_{2}$. As $m_{1} \geqslant 1$ and $m_{2} \geqslant 1$, we have $\left(m_{1}-1\right)\left(m_{2}-1\right) \geqslant 0$, which implies $m_{1} m_{2} \geqslant m_{1}+m_{2}-1$. Similarly $n_{1} n_{2} \geqslant n_{1}+n_{2}-1$. Thus

$$
\omega=m_{1} m_{2} n_{1} n_{2} \geqslant\left(m_{1}+m_{2}-1\right)\left(n_{1}+n_{2}-1\right) \geqslant\left(m_{1}+m_{2}-1\right)^{2} .
$$

Suppose that $m_{1}+m_{2} \geqslant \frac{e}{r}$. Then

$$
\omega \geqslant\left(m_{1}+m_{2}-1\right)^{2} \geqslant\left(\frac{e}{r}-1\right)^{2}=\frac{(e-r)^{2}}{r^{2}} .
$$

Since $e \geqslant r^{2}+1$ and $r \geqslant 2$, we obtain

$$
(e-r)^{2}-r(r-1)(e-r+1)=\left(e-r^{2}-1\right)\left(r^{2}-r+2\right)+1 \geqslant 0 \cdot 0+1 .
$$

Hence $\omega>\frac{(r-1)(e-r+1)}{r}$ if $m_{1}+m_{2} \geqslant \frac{e}{r}$. Thus to find the value of $\bar{\omega}$, we may assume that $m_{1}+m_{2}<\frac{e}{r}$.

Fix $m_{1}, m_{2}$ that satisfy the conditions

$$
m_{1} \geqslant 1, m_{2} \geqslant 1, \frac{e}{r}>m_{1}+m_{2} \geqslant r .
$$

Now let us find the minimum of $n_{1} n_{2}$ subject to $n_{1} \geqslant 1, n_{2} \geqslant 1$ and (5.1). The constraint (5.1) is equivalent to (5.3) with $a=m_{1}+m_{2}, b=m_{1}, x=n_{1}$ and $y=n_{2}$. Also $a+b=2 m_{1}+m_{2}<r\left(m_{1}+m_{2}\right) \leqslant e$. Since $a>b$, Lemma 5.2 implies that $n_{1} n_{2}$ is at a minimum when

$$
n_{2}=1, n_{1}:=n_{1}\left(e, m_{1}, m_{2}\right)=\frac{e-b}{a}=\frac{e-m_{1}}{m_{1}+m_{2}} .
$$

Clearly

$$
n_{1}\left(e, m_{1}, m_{2}\right)>\frac{e-\left(m_{1}+m_{2}\right)}{m_{1}+m_{2}}>\frac{e-\frac{e}{r}}{\frac{e}{r}}=r-1 \geqslant 1,
$$

and hence $n_{1}\left(e, m_{1}, m_{2}\right)+1 \geqslant r$. Thus the problem of minimizing $m_{1} m_{2} n_{1} n_{2}$ subject to (5.1) and (5.6) is equivalent to the problem

$$
\text { minimize } \tau:=m_{1} m_{2} n_{1}\left(e, m_{1}, m_{2}\right)=\frac{\left(e-m_{1}\right) m_{1} m_{2}}{m_{1}+m_{2}} \text { subject to (5.9). }
$$

Fix $m_{1}$. Since the function $\frac{t}{m_{1}+t}$ increases for $t>0$, the minimum of $\tau$ in (5.11) is achieved only in the following two cases: 
1. Case 1: $m_{2}=r-m_{1}$ if $1 \leqslant m_{1} \leqslant r-1$;

2. Case 2: $m_{2}=1$ if $m_{1} \geqslant r-1$.

Consider first Case 1. Assume first that $r=2$. Then $m_{1}=m_{2}=1$, and the minimum of $\tau$ subject to Case 1 is $\frac{(e-1)}{2}$.

Now assume that $r \geqslant 3$. Then $\tau=f\left(m_{1}\right)$, where $f(t):=\frac{t(r-t)(e-t)}{r}$. Note that $f(t)$ is a cubic with zeros at $0, r$ and $e$. Also $f(t)<0$ for $t<0$ and $f(t)>0$ for $t>e$. Hence $f^{\prime}(t)=0$ for some $t=t_{1} \in(0, r)$ and some $t=t_{2} \in(r, e)$. Note that $f(t)$ increases on $\left(-\infty, t_{1}\right)$ and decreases on $\left(t_{1}, t_{2}\right)$.

We need to find $\min _{t \in[1, r-1]} f(t)$. Clearly

$$
r f^{\prime}(t)=(r-t)(e-t)-t(e-t)-t(r-t)=(r-2 t)(e-2 t)-t^{2} .
$$

As $(r-2)(e-2) \geqslant e-2 \geqslant r^{2}-1 \geqslant 8$, we deduce that $f^{\prime}(1)>0$. As $r-2(r-1)=2-r<0$ and $(e-2 r+2) \geqslant\left(r^{2}-2 r+3\right)=(r-1)^{2}+2>0$, it follows that $f^{\prime}(r-1)<0$. So $1<t_{1}<r-1$. Hence

$$
\min _{t \in[1, r-1]} f(t)=\min (f(1), f(r-1))=f(r-1)=\frac{(e-r+1)(r-1)}{r},
$$

which is achieved only for $m_{1}=r-1$ and $m_{2}=1$.

We now consider Case 2. In this case $\tau=g\left(m_{1}\right)$, where

$$
g(t)=\frac{(e-t) t}{t+1}=e+1-t-\frac{e+1}{t+1} .
$$

Thus on $[0, \infty)$, the function $g^{\prime}(t)$ vanishes at the point

$$
t_{0}=\sqrt{e+1}-1>\sqrt{r^{2}}-1=r-1 .
$$

Note that $t_{0}$ is the unique solution of (5.1), where $m_{1}=n_{1}=t$ and $m_{2}=n_{2}=1$. So $f(t)$ increases on $\left[0, t_{0}\right]$ and decreases on the interval $\left[t_{0}, \infty\right)$. Our assumption that $m_{1}+m_{2}=m_{1}+1 \leqslant n_{1}+n_{2}=n_{1}+1$ is equivalent to $m_{1} \leqslant n_{1}$. So $m_{1} \leqslant t_{0} \leqslant n_{1}$. The assumption that $m_{1}+m_{2}=m_{1}+1 \geqslant r$ means that $m_{1} \geqslant r-1$. Hence $g\left(m_{1}\right)$ has a unique minimum at $m_{1}=r-1$. This corresponds to $n_{1}=\frac{e-r+1}{r}$. Interchanging $\left(m_{1}, m_{2}\right)$ with $\left(n_{1}, n_{2}\right)$ we obtain the second solution $\left(m_{1}, m_{2}\right)=\left(\frac{e-r+1}{r}, 1\right),\left(n_{1}, n_{2}\right)=(r, 1)$.

Theorem 5.5. Suppose one of the following conditions holds.

1. $r=2, e \geqslant 3$ is odd and $2 \leqslant p \leqslant q, l=\frac{e-1}{2}<q$.

2. $3 \leqslant r \in \mathbb{N}$ and (5.5) holds.

Then $\min _{G \in \mathcal{K}_{2}(p, q, e)} \lambda_{\text {max }}(G)$ is achieved only for $G_{D}$ isomorphic to the graph obtained from $K_{r-1, l+1}$ by adding one vertex to the group of $r-1$ vertices and connecting it to $l$ vertices in the group of $l+1$ vertices.

Proof. Assume that $G_{D}$ has the Ferrers diagram given in Figure 2 with $h=2$. Let $n_{1}=r_{2}$ and $n_{2}=r_{1}-r_{2}$. Assume first that $r=2$ and $3 \leqslant e$ is odd. Then $m_{1} \geqslant 1, m_{2} \geqslant 1$, $n_{1} \geqslant 1$ and $n_{2} \geqslant 1$, so $m_{1}+m_{2} \geqslant 2$ and $n_{1}+n_{2} \geqslant 2$. Then for $e \geqslant 5$ the theorem follows from Theorem 5.4 for $r=2$ and Theorem 4.1. For $e=3$ the theorem is trivial. For $r \geqslant 3$ the theorem follows from Proposition 5.3, Theorem 5.4 and Theorem 4.1. 


\section{$6 \quad$ A special case of Problem 5.1}

We now discuss a special case of Problem 5.1 that is not covered by Theorem 5.4.

Theorem 6.1. Let $e=3 k+1$, where $k \geqslant 7$ is an integer. Consider the minimum of $\omega=m_{1} m_{2} n_{1} n_{2}$, where $m_{1}, m_{2}, n_{1}$ and $n_{2}$ are positive integers satisfying the constraints $m_{1}\left(n_{1}+n_{2}\right)+m_{2} n_{1}=e, m_{1}+m_{2} \geqslant 3, n_{1}+n_{2} \geqslant 3$; in other words, (5.1) and (5.6) with $r=3$ hold. Then the minimum of $\omega$ is $2 k$, and it is achieved if and only if one of the following cases holds:

$$
\begin{aligned}
& \left(m_{1}, m_{2}\right)=(1,2),\left(n_{1}, n_{2}\right)=(k, 1) \\
& \left(m_{1}, m_{2}\right)=(k, 1),\left(n_{1}, n_{2}\right)=(1,2) .
\end{aligned}
$$

Proof. Clearly, if (6.1) or (6.2) holds, then $\omega=2 k$. Thus it is enough to show that for all integer values of $m_{1}, m_{2}, n_{1}$ and $n_{2}$ satisfying the constraints that are different from the values given in (6.1) and (6.2), we have $\omega>2 k$.

Since we can interchange $m_{1}$ with $n_{1}$ and $m_{2}$ with $n_{2}$, we assume without loss of generality that $n_{1}+n_{2} \geqslant m_{1}+m_{2}$. We denote the product $m_{1} m_{2}$ by $X$. Since $m_{1}+m_{2} \geqslant 3$, it follows that $X \geqslant 2$.

Case 1: $m_{1}+m_{2} \geqslant k$. Since $n_{1}+n_{2} \geqslant m_{1}+m_{2} \geqslant k$, we have $m_{1} m_{2} \geqslant k-1$ and $n_{1} n_{2} \geqslant k-1$. This implies $\omega=m_{1} m_{2} n_{1} n_{2} \geqslant(k-1)^{2}>2 k$ since $k \geqslant 7$.

Case 2: $3<m_{1}+m_{2}<k$. Hence $X \geqslant 3$. Suppose $X \geqslant k-1$. Since $n_{1}+n_{2} \geqslant 4$, we have $n_{1} n_{2} \geqslant 3$. Thus $\omega=m_{1} m_{2} n_{1} n_{2} \geqslant 3(k-1)>2 k$ since $k \geqslant 7$.

So for the remaining part of Case 2 we assume that $X<k-1$, and hence $\left\lfloor\frac{2 k}{X}\right\rfloor \geqslant 2$. We will now show that $n_{1} n_{2} \geqslant\left\lfloor\frac{2 k}{X}\right\rfloor+1$, from which it will follow that $\omega=X n_{1} n_{2}>X \frac{2 k}{X}=2 k$, as required.

Assume to the contrary that $n_{1} n_{2}<\left\lfloor\frac{2 k}{X}\right\rfloor+1$. Since $n_{1} n_{2} \leqslant\left\lfloor\frac{2 k}{X}\right\rfloor$ we have $n_{1}+n_{2} \leqslant$ $\left\lfloor\frac{2 k}{X}\right\rfloor+1$, and hence $n_{1} \leqslant\left\lfloor\frac{2 k}{X}\right\rfloor$. We now obtain an upper bound for $e$.

Clearly

$$
e=m_{1}\left(n_{1}+n_{2}\right)+m_{2} n_{1} \leqslant m_{1}\left(\left\lfloor\frac{2 k}{X}\right\rfloor+1\right)+m_{2} n_{1} \leqslant m_{1}\left(\left\lfloor\frac{2 k}{X}\right\rfloor+1\right)+m_{2}\left\lfloor\frac{2 k}{X}\right\rfloor .
$$

Observe that that for any $0<a \in \mathbb{R}$ we have the inequality

$$
m_{1}(a+1)+m_{2} a \leqslant m_{1} m_{2}(a+1)+a=X(a+1)+a
$$

and hence

$$
e \leqslant X\left(\left\lfloor\frac{2 k}{X}\right\rfloor+1\right)+\left\lfloor\frac{2 k}{X}\right\rfloor=X\left\lfloor\frac{2 k}{X}\right\rfloor+X+\left\lfloor\frac{2 k}{X}\right\rfloor .
$$

Let $f(x)=x+\frac{2 k}{x}$. Since $f$ is strictly convex on $(0, \infty)$, it follows that $f(X) \leqslant$ $\max (f(3), f(k-2))=\max \left(3+\frac{2 k}{3}, k-2+\frac{2 k}{k-2}\right)$. Hence $f(X)<k+1$ for $k>6$. We also note that in the range $[3, k-2], f(x)$ has a unique minimum at $x=\sqrt{2 k}$. Furthermore, $f(x)$ is strictly increasing after that point.

Consider the function $g(x)=x+\left\lfloor\frac{2 k}{x}\right\rfloor$ on the interval $x \in(0, \infty)$. Note that $g(x)$ is piecewise linear, where each linear piece has slope 1 , and is continuous from the left, with 
jumps at $x_{i}=\frac{2 k}{i}$ for $i \in \mathbb{N}$. So $g\left(x_{i}\right)=f\left(x_{i}\right)$ for $i \in N$, and $g(x)<f(x)$ if $0<x \neq x_{i}$ for $i \in N$. This implies $g(X)=X+\left\lfloor\frac{2 k}{X}\right\rfloor \leqslant f(X)<k+1$ for $k>6$. Since $g(X)$ is an integer, we deduce that $g(X) \leqslant k$ when $k>6$ and $X \in[3, k-2]$. Use (6.3) to deduce that

$$
e \leqslant X\left\lfloor\frac{2 k}{X}\right\rfloor+g(X) \leqslant X \frac{2 k}{X}+g(X) \leqslant 2 k+k=3 k .
$$

This contradicts the assumption that $e=3 k+1$, and completes Case 2 .

Case 3: $m_{1}+m_{2}=3$. Then $m_{1} m_{2}=2$ and $e=3 n_{1}+m_{1} n_{2}$. Assume first that $m_{1}=2$ and $m_{2}=1$. Since $e=3 k+1$ it follows that $n_{2} \geqslant 2$. So $3 n_{1} n_{2}=n_{2}\left(e-2 n_{2}\right)$. Since $e \geqslant 22$, the minimum of $n_{1} n_{2}$ is achieved either for $n_{2}=2$ or for the maximum possible value of $n_{2}$ obtained when $n_{1}=1$. For $n_{2}=2$ we have $n_{1}=k-1$ and $\omega=4(k-1)>2 k$ if $k>2$. For $n_{1}=1$ we have $n_{2}=\frac{e-3}{2}$, which may not be an integer, and $\omega=(e-3)=3 k-2>2 k$ for $k>2$.

Assume finally that $m_{1}=$ and $m_{2}=2$. Then $e=3 n_{1}+n_{2}$. Lemma 5.2 yields that $n_{1} n_{2} \geqslant \frac{e-1}{3}=k$, and equality holds if and only if $n_{2}=1$ and $n_{1}=k$. This completes Case 3 and the proof of the theorem.

We used software to show that Theorem 6.1 holds for $k=2,3,4,5,6$.

\section{C-matrices}

Let $\mathbb{R}_{+\searrow}^{p}:=\left\{\mathbf{c}=\left(c_{1}, \ldots, c_{p}\right) \in \mathbb{R}^{p}, c_{1} \geqslant \cdots \geqslant c_{p} \geqslant 0\right\}$. With each $\mathbf{c} \in \mathbb{R}_{+\searrow}^{p}$ we associate the following symmetric matrix.

$$
M(\mathbf{c})=\left[c_{\min (i, j)}\right]_{i, j=1}^{p} .
$$

The following result is well-known [4, §3.3, pp.110-111].

Proposition 7.1. Let $\mathbf{c}=\left(c_{1}, \ldots, c_{p}\right) \in \mathbb{R}_{+\searrow}^{p}$. Then all the minors of $M(\mathbf{c})$ are nonnegative. In particular $M(\mathbf{c})$ is a nonnegative definite matrix. If $c_{1}>\cdots>c_{p}>0$, then all the principal minors of $M(\mathbf{c})$ are positive, i.e., $M(\mathbf{c})$ is positive definite.

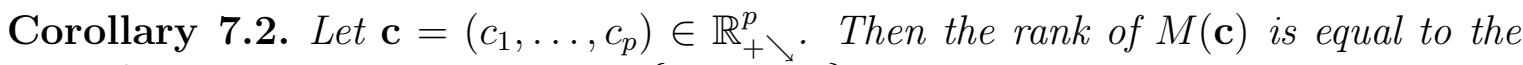
number of distinct positive elements in $\left\{c_{1}, \ldots, c_{p}\right\}$.

Proof. Let $\left\{c_{i_{1}}, \ldots, c_{i_{k}}\right\}$ be the set of all distinct positive elements in $\left\{c_{1}, \ldots, c_{p}\right\}$. Hence the rank of $M(\mathbf{c})$ is at most $k$. Let $F$ be the principal submatrix of $M(\mathbf{c})$ based on the rows and columns $\left\{i_{1}, \ldots, i_{k}\right\}$. Proposition 7.1 yields that rank $F=k$.

In what follows we assume that $\mathbf{c}=\left(c_{1}, \ldots, c_{p}\right) \in \mathbb{R}_{+\searrow}^{p}$ unless stated otherwise. Assume that $c_{1} \geqslant \cdots \geqslant c_{m}>0=c_{m+1}=\cdots=c_{p}$. Denote by $\mathbf{c}_{+}:=\left(c_{1}, \ldots, c_{m}\right)$. Then $M\left(\mathbf{c}_{+}\right)$is the principal submatrix of $M(\mathbf{c})$ obtained from $M(\mathbf{c})$ by deleting the last $p-m$ zero rows and columns. Let

$$
\lambda_{1}(\mathbf{c}) \geqslant \lambda_{2}(\mathbf{c}) \geqslant \cdots \geqslant \lambda_{p}(\mathbf{c}) \geqslant 0
$$


be the $p$ eigenvalues of $M(\mathbf{c})$. Let $m^{\prime}$ be the number of distinct elements in $\left\{c_{1}, \ldots, c_{m}\right\}$. Corollary 7.2 yields that $M(\mathbf{c})$ has exactly $m^{\prime}$ positive eigenvalues and $\lambda_{i}(\mathbf{c})=\lambda_{i}\left(\mathbf{c}_{+}\right)$for $i=1, \ldots, m$.

Let $\mathrm{S}_{m}(\mathbb{R})$ be the space of $m \times m$ real symmetric matrices. Since $\lambda_{1}(M)$ is a convex function on $S_{m}(\mathbb{R})$ by

$$
\max _{\|\mathbf{z}\|=1} \mathbf{z}^{\top} \frac{A+B}{2} \mathbf{z} \leqslant \frac{\max _{\|\mathbf{x}\|=1} \mathbf{x}^{\top} A \mathbf{x}+\max _{\|\mathbf{y}\|=1} \mathbf{y}^{\top} B \mathbf{y}}{2},
$$

we obtain the following result.

Proposition 7.3. Let $\mathrm{C} \subset \mathbb{R}_{+\backslash}^{p}$ be a compact convex set. Let $\mathcal{E}(\mathrm{C})$ be the set of the extreme points of $\mathrm{C}$. Then $\max _{\mathbf{c} \in \mathrm{C}} \lambda_{1}(\mathbf{c})=\max _{\mathbf{c} \in \mathcal{E}(C)} \lambda_{1}(\mathbf{c})$.

We show that $\lambda_{1}\left(G_{D}\right)=\lambda_{1}(\mathbf{d})$ for a corresponding vector $\mathbf{d} \in \mathbb{R}_{+\searrow}^{p}$. Let $D=$ $\left\{d_{1} \geqslant d_{2} \geqslant \cdots \geqslant d_{m}\right\}$ be a set of positive integers. Assume that $m \leqslant p$ and let

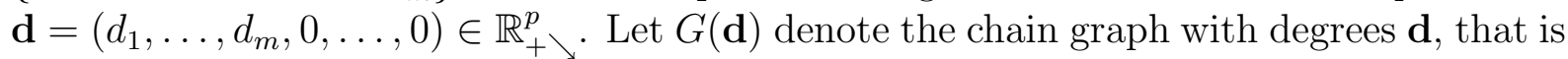
to say the chain graph $G_{D}$ with $p-m$ additional isolated vertices, where $D=\left\{d_{1}, \ldots, d_{m}\right\}$. Let $A(\mathbf{d})$ be the representation matrix of $G(\mathbf{d})$. Note that $A\left(\mathbf{d}_{+}\right)$is the representation matrix of $G_{D}$. Clearly $A(\mathbf{d}) A(\mathbf{d})^{\top}=M(\mathbf{d})$. Hence

$$
\lambda_{\max }\left(G_{D}\right)^{2}=\lambda_{1}(\mathbf{d})=\lambda_{1}\left(\mathbf{d}_{+}\right) .
$$

Thus $M(\mathbf{d})$ can be viewed as a continuous version of $G(\mathbf{d})$. The main idea of the proof of Conjecture 1.2, under the conditions discussed in the Introduction, is to replace the maximum discussed in Problem 1.1 with the maximization problem discussed in Proposition 7.3 with a carefully chosen $\mathrm{C}$.

We now bring a few inequalities for $\lambda_{1}(\mathbf{d})$ needed later, which can be viewed as a generalizations of Proposition 2.1 and Theorem 4.1.

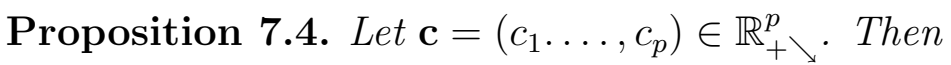

$$
\begin{aligned}
& e(\mathbf{c}):=\operatorname{trace} M(\mathbf{c})=\sum_{i=1}^{p} c_{i}=\sum_{i=1}^{p} \lambda_{i}(\mathbf{c}), \\
& \sum_{i=1}^{p} \lambda_{i}(\mathbf{c})^{2}=\operatorname{trace} M(\mathbf{c})^{2}=\sum_{i=1}^{p}(2 i-1) c_{i}^{2}, \\
& \sum_{1 \leqslant i<j \leqslant p} \lambda_{i}(\mathbf{c}) \lambda_{j}(\mathbf{c})=\sum_{1 \leqslant i<j \leqslant p} c_{j}\left(c_{i}-c_{j}\right) .
\end{aligned}
$$

Hence $\lambda_{1}(\mathbf{c}) \leqslant e(\mathbf{c})$. Equality holds if and only if $M(\mathbf{c})$ has rank one. Moreover,

$$
\lambda_{1}(\mathbf{c}) \leqslant \sqrt{\sum_{i=1}^{p}(2 i-1) c_{i}^{2}} .
$$


A sharper upper estimate of $\lambda_{1}(\mathbf{c})$ for $p \geqslant 2$ is given as follows. Assume that the set $\left\{c_{1}, \ldots, c_{p}\right\}$ consists of $h \geqslant 2$ distinct positive numbers. Then

$$
\lambda_{1}(\mathbf{c}) \leqslant \frac{\left(2 \alpha_{h}-1\right) e(\mathbf{c})+\sqrt{e(\mathbf{c})^{2}-4 \alpha_{h} \beta}}{2 \alpha_{h}},
$$

where

$$
\alpha_{h}=\frac{h}{2(h-1)}, \quad \beta=\sum_{1 \leqslant i<j \leqslant p} c_{j}\left(c_{i}-c_{j}\right) .
$$

For a fixed $\beta$, the right-hand side of (7.7) is an increasing sequence for $h=2,3, \ldots$, and its limit as $h \rightarrow \infty$ is the right-hand side of (7.6).

Proof. The equalities (7.3), (7.4) are straightforward. The equality (7.5) follows from them and the identity

$$
2 \sum_{1 \leqslant i<j \leqslant p} \lambda_{i} \lambda_{j}=\left(\sum_{i=1}^{p} \lambda_{i}\right)^{2}-\sum_{i=1}^{p} \lambda_{i}^{2} .
$$

Since all $\lambda_{i}(\mathbf{c})$ are real, the inequality (7.6) follows from (7.4).

We now show how the estimate (7.7) follows from (7.5). By Corollary 7.2 the rank of $M(\mathbf{c})$ is exactly $h$, and therefore exactly $h$ eigenvalues of $M(\mathbf{d})$ are positive. Thus in the left-hand side of the equalities (7.3)-(7.5), $i$ and $j$ can run from 1 to $h$ only. For $h=2$, (7.7) follows with equality from (7.3) and (7.5).

Assume that $h \geqslant 3$. We let $\lambda_{1}(\mathbf{c})=x$ and $e(\mathbf{c})=e$ and rewrite (7.5) as follows:

$$
x(e-x)=\beta-\sum_{2 \leqslant i<j \leqslant h} \lambda_{i}(\mathbf{c}) \lambda_{j}(\mathbf{c}) .
$$

In (7.9) we are free to choose $\lambda_{2}(\mathbf{c}), \ldots, \lambda_{h}(\mathbf{c})$ subject to $x+\sum_{2 \leqslant i \leqslant h} \lambda_{i}(\mathbf{c})=e$, and wish to maximize $x$.

Observe that the right-hand side of (7.9) is always nonnegative by (7.5) and the definition of $\beta$. Thus (7.9) has two solutions $x$ between 0 and $e$, and we are interested in the larger one and want to maximize it. This is equivalent to choosing $\lambda_{2}(\mathbf{c}), \ldots, \lambda_{h}(\mathbf{c})$ so as to minimize the right-hand side of (7.9), or equivalently to maximize $\sum_{2 \leqslant i<j \leqslant h} \lambda_{i}(\mathbf{c}) \lambda_{j}(\mathbf{c})$. It is well-known that a sum of the form $\sum_{1 \leqslant i<j \leqslant k} a_{i} a_{j}$ can only increase if the $a_{i}$ are each replaced by their arithmetic mean $\frac{\sum a_{i}}{k}$. Indeed, $\left(\sum 1 \cdot a_{i}\right)^{2} \leqslant\left(\sum 1^{2}\right)\left(\sum a_{i}^{2}\right)=$ $k \sum a_{i}^{2}$. Therefore $2 \sum_{\mathbf{i}<j} a_{i} a_{j}=\left(\sum a_{i}\right)^{2}-\sum a_{i}^{2} \leqslant\left(\sum a_{i}\right)^{2}-\frac{1}{k}\left(\sum a_{i}\right)^{2}=\left(\sum a_{i}\right)^{2} \frac{k-1}{k}=$ $2\left(\begin{array}{c}k \\ 2\end{array}\right)\left(\frac{\sum a_{i}}{k}\right)^{2}$.

Thus the upper estimate on $x$ is achieved if we set each of $\lambda_{2}(\mathbf{c}), \ldots, \lambda_{h}(\mathbf{c})$ equal to $y$. Then $x$ and $y$ are subject to $x+(h-1) y=e$ and $x(e-x)+\left(\begin{array}{c}h-1 \\ 2\end{array}\right) y^{2}=\beta$. Eliminating $y$, we see that $x$ should satisfy

$$
x(e-x)+\frac{h-2}{2(h-1)}(e-x)^{2}=\beta,
$$


and the larger solution of (7.10) yields (7.7) for $h \geqslant 3$.

The left-hand side of (7.10) is a quadratic in $x$, which is positive for $0<x<e$ and increases with $h$. Therefore the larger solution of (7.10) increases with $h$. When we take (7.10) to the limit $h \rightarrow \infty$, we obtain $x(e-x)+\frac{1}{2}(e-x)^{2}=\beta$, or equivalently

$$
x^{2}=e^{2}-2 \beta=\sum_{i=1}^{p}(2 i-1) c_{i}^{2},
$$

and the positive solution of (7.11) is equal to the right-hand side of (7.6).

\section{A proof of Conjecture 1.2 in certain cases}

Theorem 8.1. Let $2 \leqslant r \leqslant l$ be two positive integers. Assume that $e=r l+r-1$. Suppose one of the following conditions holds:

1. $r=2$ and $2 \leqslant p \leqslant q, l=\frac{e-1}{2}<q$;

2. $3 \leqslant r \leqslant l$ and $r \leqslant p \leqslant l+1 \leqslant q \leqslant l+1+\frac{l}{r-1}$.

Let $G_{r, l+1}$ be the graph obtained from $K_{r-1, l+1}$ by adding one vertex to the group of $r-1$ vertices and connecting it to $l$ vertices in the group of $l+1$ vertices. Then $\lambda_{\max }(G) \leqslant$ $\lambda_{\text {max }}\left(G_{r, l+1}\right)$, for all $G \in \mathcal{K}(p, q, e)$. Equality holds if and only if $G$ is isomorphic to $G_{r, l+1}$.

Proof. Corollary 3.6 implies that in order to find $\max _{G \in \mathcal{K}(p, q, e)}$, it is enough to consider graphs $G_{D}=(U, V, E)$, for some $D=\left\{d_{1} \geqslant d_{2} \geqslant \cdots \geqslant d_{m}\right\}$, where $m \leqslant p$ and $d_{1} \leqslant q$. We are going to assume that $\# U=m \leqslant \# V=d_{1}$ (if this is not satisfied consider the isomorphic graph $\left.G_{D}^{\prime}=(V, U, E)\right)$. Let $\mathbf{d}=\left(d_{1}, \ldots, d_{m}\right)$ be the degree sequence of $D$.

Since $\mathcal{K}(e, p, q)$ does not contain a complete bipartite graph, we know that $m \geqslant 2$. Proposition 5.3 yields that $m \geqslant r$ for $r \geqslant 3$. Let $\delta_{i}:=d_{i}-d_{m}$ for $i=1, \ldots, m$. We define $s$ by $\delta_{s}>0=\delta_{s+1}=\cdots=\delta_{m}$, and put $\boldsymbol{\delta}:=\left(\delta_{1}, \ldots, \delta_{s}\right)^{\top}$. Thus switching from $\mathbf{d}$ to $\boldsymbol{\delta}$ amounts to deleting the first $d_{m}$ columns of the Ferrers diagram of $\mathbf{d}$, and then deleting empty rows. The resulting Ferrers diagram has a total of $e^{\prime}:=e-m d_{m}=\sum_{i=1}^{s} \delta_{i}$ components equal to 1 and the rest are zero. Note that $\delta_{1} \leqslant q-d_{m}$. As $h \geqslant 2$, we have that $\delta_{s} \geqslant 1$. Hence $e^{\prime} \leqslant e-1$ and $\frac{e^{\prime}}{s} \geqslant 1$.

We now consider the following polyhedron in $\mathbb{R}^{s}$ :

$$
P:=\left\{\left(x_{1}, \ldots, x_{s}\right)^{\top} \in \mathbb{R}^{s}, \quad x_{1} \geqslant x_{2} \geqslant \cdots \geqslant x_{s} \geqslant 0, \quad \sum_{i=1}^{s} x_{i}=e^{\prime}\right\} .
$$

Using the notation

$$
\mathbf{1}_{n, i}:=(\underbrace{1, \ldots, 1}_{i}, 0, \ldots, 0)^{\top} \in \mathbb{R}^{n}, \quad i=1, \ldots, n,
$$

it is clear that the extreme points of $P$ are $\frac{e^{\prime}}{i} \mathbf{1}_{s, i}, i=1, \ldots, s$. 
Let us define

$$
\mathbf{a}_{i}(\mathbf{d})=\left(a_{1, i}, \ldots, a_{m, i}\right)^{\top}:=\frac{e^{\prime}}{i} \mathbf{1}_{m, i}+d_{m} \mathbf{1}_{m, m}, \quad i=1, \ldots, s .
$$

We note that $\mathbf{d} \in \mathrm{C}(\mathbf{d}):=\operatorname{conv}\left\{\mathbf{a}_{1}(\mathbf{d}), \ldots, \mathbf{a}_{s}(\mathbf{d})\right\}$. Indeed, $\boldsymbol{\delta} \in P$, and therefore there exist $\alpha_{1}, \ldots, \alpha_{s} \geqslant 0$ satisfying $\sum_{i=1}^{s} \alpha_{i}=1$ and $\sum_{i=1}^{s} \alpha_{i} \frac{e_{i}^{\prime}}{\mathbf{1}_{s, i}}=\boldsymbol{\delta}$. Then

$$
\begin{array}{r}
\sum_{i=1}^{s} \alpha_{i} \mathbf{a}_{i}(\mathbf{d})=\sum_{i=1}^{s} \alpha_{i}\left(\frac{e^{\prime}}{i} \mathbf{1}_{m, i}+d_{m} \mathbf{1}_{m, m}\right)=\left(\sum_{i=1}^{s} \alpha_{i} \frac{e^{\prime}}{i} \mathbf{1}_{m, i}\right)+d_{m} \mathbf{1}_{m, m} \\
\left(d_{1}, \ldots, d_{s}, d_{m}, \ldots, d_{m}\right)^{\top}=\left(d_{1}, \ldots, d_{s}, d_{s+1}, \ldots, d_{m}\right)^{\top}=\mathbf{d} .
\end{array}
$$

Since $\mathbf{d}$ is a convex combination of $\mathbf{a}_{1}(\mathbf{d}), \ldots, \mathbf{a}_{s}(\mathbf{d})$, it follows that $M(\mathbf{d})$ is the same convex combination of $M\left(\mathbf{a}_{1}(\mathbf{d})\right), \ldots, M\left(\mathbf{a}_{s}(\mathbf{d})\right)$. Combine (7.2) with Proposition 7.3 to obtain

$$
\lambda_{\max }\left(G_{D}\right)^{2}=\lambda_{1}(M(\mathbf{d})) \leqslant \max _{1 \leqslant k \leqslant l} \lambda_{1}\left(M\left(\mathbf{a}_{k}(\mathbf{d})\right)\right) .
$$

The vector $\mathbf{a}_{k}(\mathbf{d})$ has the form $\mathbf{a}_{k}(\mathbf{d})=(\underbrace{x, \ldots, x}_{k}, \underbrace{y, \ldots, y}_{m-k})^{\top}$ with $x=\frac{e^{\prime}}{k}+d_{m}$ and $y=d_{m}$. Therefore the first $k$ rows of $M\left(\mathbf{a}_{k}(\mathbf{d})\right)$ are equal to $\mathbf{a}_{k}(\mathbf{d})$ and the last $m-k$ rows are equal to $y \mathbf{1}_{m, m}$. Proposition 7.1 and Corollary 7.2 yield that $M\left(\mathbf{a}_{k}(\mathbf{d})\right)$ is a nonnegative definite matrix of rank 2 . It satisfies

$$
\lambda_{1}\left(M\left(\mathbf{a}_{k}(\mathbf{d})\right)\right)+\lambda_{2}\left(M\left(\mathbf{a}_{k}(\mathbf{d})\right)\right)=\operatorname{trace} M\left(\mathbf{a}_{k}(\mathbf{d})\right)=k\left(\frac{e^{\prime}}{k}+d_{m}\right)+(m-k) d_{m}=e .
$$

The product $\lambda_{1}\left(M\left(\mathbf{a}_{k}(\mathbf{d})\right)\right) \lambda_{2}\left(M\left(\mathbf{a}_{k}(\mathbf{d})\right)\right)$ is equal to the sum

$$
\sum_{i<j} \lambda_{i}\left(M\left(\mathbf{a}_{k}(\mathbf{d})\right)\right) \lambda_{j}\left(M\left(\mathbf{a}_{k}(\mathbf{d})\right)\right)
$$

which in turn equals the coefficient of $\lambda^{m-2}$ in the characteristic polynomial

$$
\prod_{i}\left(\lambda-\lambda_{i}\left(M\left(\mathbf{a}_{k}(\mathbf{d})\right)\right)\right)
$$

This coefficient equals in turn the sum of all $2 \times 2$ principal minors of $M\left(\mathbf{a}_{k}(\mathbf{d})\right)$. There are $k(m-k)$ contributing minors, each of the form $\operatorname{det}\left(\begin{array}{ll}x & y \\ y & y\end{array}\right)=y(x-y)$. Therefore

$$
\lambda_{1}\left(M\left(\mathbf{a}_{k}(\mathbf{d})\right)\right) \lambda_{2}\left(M\left(\mathbf{a}_{k}(\mathbf{d})\right)\right)=\omega\left(\mathbf{a}_{k}(\mathbf{d})\right):=k(m-k) \frac{e^{\prime}}{k} d_{m}
$$

Hence

$$
\lambda_{1}\left(M\left(\mathbf{a}_{k}(\mathbf{d})\right)\right)=\frac{e+\sqrt{e^{2}-4 \omega\left(a_{k}(\mathbf{d})\right)}}{2} .
$$


Thus the maximum possible value of $\lambda_{1}\left(M\left(\mathbf{a}_{k}(\mathbf{d})\right)\right)$ is achieved for the minimum value of $\omega\left(\mathbf{a}_{k}(\mathbf{d})\right)$. This situation corresponds to the minimization problem we studied in Theorem 5.4. We put

$$
m_{1}=k, \quad m_{2}=m-k, \quad n_{1}=d_{m}, \quad n_{2}=\frac{e^{\prime}}{k} .
$$

Clearly $m_{1} \geqslant 1$ and $n_{1} \geqslant 1$. Also $m_{2}=m-k \geqslant s+1-k \geqslant 1$ and $n_{2}=\frac{e^{\prime}}{k} \geqslant \frac{e^{\prime}}{s} \geqslant 1$. Recall that $m=m_{1}+m_{2} \geqslant r$. We claim that for each $2 \leqslant r \in \mathbb{N}$, the inequality $n_{1}+n_{2} \geqslant r$ holds. For $r=2$ this inequality follows from the inequalities $n_{1} \geqslant 1, n_{2} \geqslant 1$. Assume now that $r \geqslant 3$. Observe that

$$
n_{1}+n_{2}=d_{m}+\frac{e^{\prime}}{k} \geqslant d_{m}+\frac{e^{\prime}}{s} \geqslant d_{m}+\frac{e^{\prime}}{m-1}=d_{m}+\frac{e-m d_{m}}{m-1}=\frac{e-d_{m}}{m-1} .
$$

Recall that $G_{D}$ is not complete bipartite. Since $d_{1} \geqslant \cdots \geqslant d_{m}$, we deduce that $d_{m} \leqslant \frac{e-1}{m}$. Hence

$$
\frac{e-d_{m}}{m-1} \geqslant f(m):=\frac{e}{m-1}+\frac{1}{m(m-1)} .
$$

So $f(m)$ is a decreasing function for $m>1$. For $m=p=l+1$, obtain $f(l+1)>\frac{e}{l}=$ $\frac{r l+r-1}{l}>r$ if $r \geqslant 3$. Hence $n_{1}+n_{2} \geqslant r$ for any $r \geqslant 2$. Theorem 5.4 implies the inequality

$$
\omega\left(\mathbf{a}_{k}(\mathbf{d})\right) \leqslant \frac{(r-1)(e-r+1)}{r} .
$$

Hence

$$
\lambda_{1}\left(M\left(\mathbf{a}_{k}(\mathbf{d})\right)\right) \leqslant \frac{e+\sqrt{e^{2}-\left(\frac{(r-1)(e-r+1)}{r}\right)^{2}}}{2}=\lambda_{\max }\left(G_{r, l+1}\right)^{2},
$$

where the last equality follows from Theorem 4.1. We use (8.4) to deduce

$$
\lambda_{\max }\left(G_{D}\right) \leqslant \lambda_{\max }\left(G_{r, l+1}\right) \text { for any } G_{D} \in \mathcal{K}(p, q, e) .
$$

It is left to show that equality holds in (8.9) if and only if $D=D_{*}:=\left\{d_{1}=\cdots=d_{r-1}=\right.$ $\left.l+1>d_{r}=l\right\}$. Let $\mathbf{d}_{*}=(l+1, \ldots, l+1,1)$ be the corresponding degree sequence of $D_{*}$. We now consider the equality case in (8.8). Theorem 5.4 asserts that equality holds only if one of the two conditions in (5.7) holds.

Assume first that the first condition of (5.7) holds. So $n_{1}=\frac{e-r+1}{r}=l$. On the other hand $n_{1}=d_{m}$. So $d_{m}=l$. Also $m=m_{1}+m_{2}=r-1+1=r$. Furthermore, $n_{2}=\frac{e^{\prime}}{k}=1$. This can happen if and only if $\delta_{1}=\cdots=\delta_{k}=1$. Hence $d_{1}=\cdots=d_{s}=l+1$ and $d_{s+1}=d_{r}=l$. Since $e=l r+r-1$, we deduce that $s=r-1$.

Assume now that the second condition holds in (8.8). So $d_{m}=n_{1}=r-1$ and $\frac{e^{\prime}}{k}=1$. Hence $d_{1}=\cdots=d_{s}=r>d_{s+1}=\cdots=d_{m}=r-1$. We have $m_{1}=\frac{e-r+1}{r}=l=k$ and $m_{2}=1$. So $m=m_{1}+m_{2}=l+1$. Since $e=r l+r-1$, we deduce that $s=r-1$. Hence $D=D^{*}=\left\{d_{1}=\cdots=d_{l}=r>d_{l+1}=r-1\right\}$. Note that $G_{D^{*}}$ is isomorphic to $G_{D_{*}}=G_{r, l+1}=\left(U_{*}, V_{*}, E\right)$. More precisely, $G_{D_{*}}=\left(V_{*}, U_{*}, E\right)$. Assume first that $r \geqslant 3$. Then $\# V_{*}=l+1>r=\# U_{*}$. This case is ruled out since we agreed to consider only 
$G_{D}=(U, V, E)$ where $\# U \leqslant \# V$. If $r=2$, then $\# V_{*}=\frac{e+1}{2}=l+1$ and $\# U=2$. If $e \geqslant 5$, then this case is ruled out as above. If $e=3$, then any $G \in \mathcal{K}(2,2,3)$ is isomorphic to $G_{2,2}$, and the theorem trivially holds in this case. In particular any $G_{D} \in \mathcal{K}(2,2,3)$ is equal to $G_{D_{*}}$.

Let $G_{D}=(U, V, E) \in \mathcal{K}(p, q, e)$, and assume that $\# U \leqslant \# V$ and $D \neq D_{*}$. The above arguments show that $\omega\left(\mathbf{a}_{k}(\mathbf{d})\right)>\omega\left(\mathbf{a}_{r-1}\left(\mathbf{d}_{*}\right)\right)$ for $k=1, \ldots, s$. Hence $\lambda_{1}\left(M\left(\mathbf{a}_{k}(\mathbf{d})\right)<\right.$ $\lambda_{\max }\left(G_{r, l+1}\right)^{2}$ for $k=1, \ldots, s$, and (8.4) yields that $\lambda_{\max }\left(G_{D}\right)<\lambda_{\max }\left(G_{r, l+1}\right)$.

\section{References}

[1] R.A. Brualdi and A.J. Hoffman, On the spectral radius of $(0,1)$-matrices, Linear Algebra Appl. 65 (1985), 133-146.

[2] S. Friedland, The maximal eigenvalue of 0-1 matrices with prescribed number of ones, Linear Algebra Appl. 69 (1985), 33-69.

[3] S. Friedland, Bounds on the spectral radius of graphs with e edges, Linear Algebra Appl. 101 (1988), 81-86.

[4] S. Karlin, Total positivity, Stanford University Press, Stanford, Calif. 1968.

[5] P. Rowlinson, On the maximal index of graphs with a prescribed number of edges, Linear Algebra Appl. 110 (1988), 43-53.

[6] F.R. Gantmacher, The Theory of Matrices Volume One, Chelsea, New York, 1977.

[7] N.V.R. Mahadev and Uri N. Peled, Threshold Graphs and Related Topics, Annals of Discrete Mathematics 56, North-Holland, New York, 1995.

[8] R.P. Stanley, A bound on the spectral radius of graphs with e edges, Linear Algebra Appl. 87 (1987), 267-269.

[9] M. Yannakakis, The complexity of the partial order dimension problem, SIAM Journal on Algebraic and Discrete Methods, 3 (1982), 351-358. 